\title{
MODULAR FORMS ON BALL QUOTIENTS OF NON-POSITIVE KODAIRA DIMENSION
}

\author{
AZNIV KASPARIAN
}

Communicated by Vasil V. Tsanov

Abstract. The Baily-Borel compactification $\widehat{B} / \Gamma$ of an arithmetic ball quotient admits projective embeddings by $\Gamma$-modular forms of sufficiently large weight. We are interested in the target and the rank of the projective map $\Phi$, determined by $\Gamma$-modular forms of weight one. This paper concentrates on the finite $H$-Galois quotients $\mathbb{B} / \Gamma_{H}$ of a specific $\mathbb{B} / \Gamma_{-1}^{(6,8)}$, birational to an abelian surface $A_{-1}$. Any compactification of $\mathbb{B} / \Gamma_{H}$ has non-positive Kodaira dimension. The rational maps $\Phi^{H}$ of $\widehat{\mathbb{B} / \Gamma_{H}}$ are studied by means of the $H$-invariant abelian functions on $A_{-1}$.

The modular forms of sufficiently large weight are known to provide projective embeddings of the arithmetic quotients of the two-ball

$$
\mathbb{B}=\left\{z=\left(z_{1}, z_{2}\right) \in \mathbb{C}^{2} ;\left|z_{1}\right|^{2}+\left|z_{2}\right|^{2}<1\right\} \simeq \mathrm{SU}(2,1) / \mathrm{S}\left(\mathrm{U}_{2} \times \mathrm{U}_{1}\right) .
$$

The present work studies the projective maps, given by the modular forms of weight one on certain Baily-Borel compactifications $\widehat{\mathbb{B} / \Gamma_{H}}$ of Kodaira dimension $\kappa\left(\widehat{\mathbb{B} / \Gamma_{H}}\right) \leq 0$. More precisely, we start with a fixed smooth Picard modular surface $A_{-1}^{\prime}=\left(\mathbb{B} / \Gamma_{-1}^{(6,8)}\right)^{\prime}$ with abelian minimal model $A_{-1}=E_{-1} \times E_{-1}$, $E_{-1}=\mathbb{C} / \mathbb{Z}+\mathbb{Z}$. Any automorphism group of $A_{-1}^{\prime}$, preserving the toroidal compactifying divisor $T^{\prime}=\left(\mathbb{B} / \Gamma_{-1}^{(6,8)}\right)^{\prime} \backslash\left(\mathbb{B} / \Gamma_{-1}^{(6,8)}\right)$ acts on $A_{-1}$ and lifts to a ball lattice $\Gamma_{H}$, normalizing $\Gamma_{-1}^{(6,8)}$. The ball quotient compactification $A_{-1}^{\prime} / H=\overline{\mathbb{B} / \Gamma_{H}}$ is birational to $A_{-1} / H$. We study the $\Gamma_{H}$-modular forms $\left[\Gamma_{H}, 1\right]$ of weight one by realizing them as $H$-invariants of $\left[\Gamma_{-1}^{(6,8)}, 1\right]$. That allows to transfer $\left[\Gamma_{H}, 1\right]$ to the $H$-invariant abelian functions, in order to determine $\operatorname{dim}_{\mathbb{C}}\left[\Gamma_{H}, 1\right]$ and the transcendence dimension of the graded $\mathbb{C}$-algebra, generated by $\left[\Gamma_{H}, 1\right]$. The last one is exactly the rank of the projective map $\Phi: \widetilde{\mathbb{B} / \Gamma_{H}} \cdots \rightarrow \mathbb{P}\left(\left[\Gamma_{H}, 1\right]\right)$. 


\section{The Transfer of Modular Forms to Meromorphic Functions is Inherited by the Finite Galois Quotients}

Definition 1. Let $\Gamma<\mathrm{SU}(2,1)$ be a lattice, i.e., a discrete subgroup, whose quotient $\mathrm{SU}(2,1) / \Gamma$ has finite invariant measure. A $\Gamma$-modular form of weight $n$ is a holomorphic function $\delta: \mathbb{B} \rightarrow \mathbb{C}$ with transformation law

$$
\gamma(\delta)(z)=\delta(\gamma(z))=[\operatorname{det} \operatorname{Jac}(\gamma)]^{-n} \delta(z) \quad \gamma \in \Gamma, \quad z \in \mathbb{B} .
$$

Bearing in mind that a biholomorphism $\gamma \in \operatorname{Aut}(\mathbb{B})$ acts on a differential form $\mathrm{d} z_{1} \wedge \mathrm{d} z_{2}$ of top degree as a multiplication by the $\operatorname{Jacobian} \operatorname{determinant} \operatorname{det} \operatorname{Jac}(\gamma)$, one constructs the linear isomorphism

$$
j_{n}:[\Gamma, n] \longrightarrow H^{0}\left(\mathbb{B},\left(\Omega_{\mathbb{B}}^{2}\right)^{\otimes n}\right)^{\Gamma}
$$

with the $\Gamma$-invariant holomorphic sections of the canonical bundle $\Omega_{\mathbb{B}}^{2}$ of $\mathbb{B}$. Thus, the graded $\mathbb{C}$-algebra of the $\Gamma$-modular forms can be viewed as the tensor algebra of the $\Gamma$-invariant volume forms on $\mathbb{B}$. For any $\delta_{1}, \delta_{2} \in[\Gamma, n]$ the quotient $\frac{\delta_{1}}{\delta_{2}}$ is a correctly defined holomorphic function on $\mathbb{B} / \Gamma$. In such a way, $[\Gamma, n]$ and $j_{n}[\Gamma, n]$ determine a projective map

$$
\Phi_{n}: \mathbb{B} / \Gamma \longrightarrow \mathbb{P}([\Gamma, n])=\mathbb{P}\left(j_{n}[\Gamma, n]\right)
$$

The $\Gamma$-cusps $\partial_{\Gamma} \mathbb{B} / \Gamma$ are of complex co-dimension two, so that $\Phi_{n}$ extends to the Baily-Borel compactification

$$
\Phi_{n}: \widehat{\mathbb{B} / \Gamma} \longrightarrow \mathbb{P}([\Gamma, n])
$$

If the lattice $\Gamma<\mathrm{SU}_{2,1}$ is torsion-free then the toroidal compactification $X^{\prime}=$ $(\mathbb{B} / \Gamma)^{\prime}$ is a smooth surface. Denote by $\rho: X^{\prime}=(\mathbb{B} / \Gamma)^{\prime} \rightarrow \widehat{X}=\widehat{\mathbb{B} / \Gamma}$ the contraction of the irreducible components $T_{i}^{\prime}$ of the toroidal compactifying divisor $T^{\prime}$ to the $\Gamma$-cusps $\kappa_{i} \in \partial_{\Gamma} \mathbb{B} / \Gamma$. The tensor product $\Omega_{X^{\prime}}^{2}\left(T^{\prime}\right)$ of the canonical bundle $\Omega_{X^{\prime}}^{2}$ of $X^{\prime}$ with the holomorphic line bundle $\mathcal{O}\left(T^{\prime}\right)$, associated with the toroidal compactifying divisor $T^{\prime}$ is the logarithmic canonical bundle of $X^{\prime}$. In [2] Hemperly has observes that

$$
H^{0}\left(X^{\prime}, \Omega_{X^{\prime}}^{2}\left(T^{\prime}\right)^{\otimes n}\right)=\rho^{*} j_{n}[\Gamma, n] \simeq[\Gamma, n] .
$$

Let $K_{X^{\prime}}$ be the canonical divisor of $X^{\prime}$

$$
\mathcal{L}_{X^{\prime}}\left(n K_{X^{\prime}}+n T^{\prime}\right)=\left\{f \in \mathfrak{M e r}\left(X^{\prime}\right) ;(f)+n K_{X^{\prime}}+n T^{\prime} \geq 0\right\}
$$


be the linear system of the divisor $n\left(K_{X^{\prime}}+T^{\prime}\right)$ and $s$ be a global meromorphic section of $\Omega_{X^{\prime}}^{2}\left(T^{\prime}\right)$. Then

$$
s^{\otimes n}: \mathcal{L}_{X^{\prime}}\left(n K_{X^{\prime}}+n T^{\prime}\right) \longrightarrow H^{0}\left(X^{\prime}, \Omega_{X^{\prime}}^{2}\left(T^{\prime}\right)^{\otimes n}\right)
$$

is a $\mathbb{C}$-linear isomorphism. Let $\xi: X^{\prime} \rightarrow X$ be the blow-down of the $(-1)$ curves on $X^{\prime}=(\mathbb{B} / \Gamma)^{\prime}$ to its minimal model $X$. The Kobayashi hyperbolicity of $\mathbb{B}$ requires $X^{\prime}$ to be the blow-up of $X$ at the singular locus $T^{\sin g}$ of $T=\xi\left(T^{\prime}\right)$. The canonical divisor $K_{X^{\prime}}=\xi^{*} K_{X}+L$ is the sum of the pull-back of $K_{X}$ with the exceptional divisor $L$ of $\xi$. The birational map $\xi$ induces an isomorphism $\xi^{*}$ : $\mathfrak{M e r}(X) \rightarrow \mathfrak{M e r}\left(X^{\prime}\right)$ of the meromorphic function fields. In order to translate the condition $\xi^{*}(f)+n K_{X^{\prime}}+n T^{\prime} \geq 0$ in terms of $f \in \mathfrak{M e r}(X)$, let us recall the notion of a multiplicity of a divisor $D \subset X$ at a point $p \in X$. If $D=\sum_{i} n_{i} D_{i}$ is the decomposition of $D$ into irreducible components then $m_{p}(D)=\sum_{i} n_{i} m_{p}\left(D_{i}\right)$, where

$$
m_{p}\left(D_{i}\right)= \begin{cases}1 & \text { for } p \in D_{i} \\ 0 & \text { for } p \notin D_{i}\end{cases}
$$

Let $L=\sum_{p \in T^{\text {sing }}} L(p)$ for $L(p)=\xi^{-1}(p)$ and $f \in \operatorname{Mer}(X)$. The condition $\xi^{*}(f)+$ $n L \geq 0$ is equivalent to $m_{p}(f)+n \geq 0$ for all $p \in T^{\operatorname{sing}}$. Thus, $\mathcal{L}_{X^{\prime}}\left(n K_{X^{\prime}}+n T^{\prime}\right)$ turns to be the pull-back of the subspace

$$
\begin{gathered}
\mathcal{L}_{X}\left(n K_{X}+n T, n T^{\mathrm{sing}}\right) \\
=\left\{f \in \mathfrak{M e r}(X) ;(f)+n K_{X}+n T \geq 0, m_{p}(f)+n \geq 0, p \in T^{\mathrm{sing}}\right\}
\end{gathered}
$$

of the linear system $\mathcal{L}_{X}\left(n K_{X}+n T\right)$. The $\mathbb{C}$-linear isomorphism

$$
\operatorname{Trans}_{n}:=\left(\xi^{*}\right)^{-1} s^{\otimes(-n)} j_{n}:[\Gamma, n] \longrightarrow \mathcal{L}_{X}\left(n K_{X}+n T, n T^{\text {sing }}\right)
$$

introduced by Holzapfel in [3], is called transfer of modular forms.

Bearing in mind Hemperly's result $H^{0}\left(X^{\prime}, \Omega_{X^{\prime}}^{2}\left(T^{\prime}\right)^{\otimes n}\right)=\rho^{*} j_{1}[\Gamma, n]$ for a fixed point free $\Gamma$, we refer to

$$
\Phi_{n}^{H}: \widehat{\mathbb{B} / \Gamma_{H}} \longrightarrow \mathbb{P}\left(\left[\Gamma_{H}, n\right]\right)=\mathbb{P}\left(j_{n}\left[\Gamma_{H}, n\right]\right)
$$

as the $n$-th logarithmic-canonical map of $\widehat{\mathbb{B} / \Gamma_{H}}$, regardless of the ramifications of $\mathbb{B} \rightarrow \mathbb{B} / \Gamma_{H}$.

The next lemma explains the transfer of modular forms on finite Galois quotients $\mathbb{B} / \Gamma_{H}$ of $\mathbb{B} / \Gamma$ to meromorphic functions on $X / H$. In general, the toroidal compactification $X_{H}^{\prime}=\left(\mathbb{B} / \Gamma_{H}\right)^{\prime}$ is a normal surface. The logarithmic-canonical bundle is not defined on a singular $X_{H}^{\prime}$, but there is always a logarithmic-canonical Weil divisor on $X_{H}^{\prime}$. 
Lemma 2. Let $A^{\prime}=(\mathbb{B} / \Gamma)^{\prime}$ be a neat toroidal compactification with an abelian minimal model $A$ and $H$ be a subgroup of $G=\operatorname{Aut}(A, T)=\operatorname{Aut}\left(A^{\prime}, T^{\prime}\right)$. Then

i) the transfer $\operatorname{Trans}_{n}:=\left(\xi^{*}\right)^{-1} s^{\otimes(-n)} j_{n}:[\Gamma, n] \longrightarrow \mathcal{L}_{A}\left(n T, n T^{\mathrm{sing}}\right)$ of $\Gamma$-modular forms to abelian functions induces a linear isomorphism

$$
\operatorname{Trans}_{n}^{H}:\left[\Gamma_{H}, n\right] \longrightarrow \mathcal{L}_{A}\left(n T, n T^{\mathrm{sing}}\right)^{H}
$$

of $\Gamma_{H}$-modular forms with rational functions on $A / H$, called also a transfer

ii) the projective maps

$$
\Phi_{n}^{H}: \widehat{\mathbb{B} / \Gamma_{H}} \cdots \mathbb{P}\left(\left[\Gamma_{H}, n\right]\right), \quad \Psi_{n}^{H}: A / H \cdots \mathbb{P}\left(\mathcal{L}_{A}\left(n T, n T^{\operatorname{sing}}\right)^{H}\right)
$$

coincide on an open Zariski dense subset.

Proof: i) Note that $j_{n}\left[\Gamma_{H}, n\right]=j_{n}[\Gamma, n]^{H}$. The inclusion $j_{n}\left[\Gamma_{H}, n\right] \subseteq j_{n}[\Gamma, n]$ follows from $\Gamma \leq \Gamma_{H}$. If $\Gamma_{H}=\cup_{j=1}^{n} \gamma_{j} \Gamma$ is the coset decomposition of $\Gamma_{H}$ modulo $\Gamma$, then $H=\left\{h_{i}=\gamma_{i} \Gamma ; 1 \leq i \leq n\right\}$. A $\Gamma$-modular form $\omega \in j_{n}[\Gamma, n]$ is $\Gamma_{H^{-}}$ modular exactly when it is invariant under all $\gamma_{i}$, which amounts to the invariance under all $h_{i}$.

One needs a global meromorphic $G$-invariant section $s$ of $\Omega_{A^{\prime}}^{2}\left(T^{\prime}\right)$, in order to obtain a linear isomorphism

$$
\left(\xi^{*}\right)^{-1} s^{\otimes(-n)}=\operatorname{Trans}_{n}^{H} j_{n}^{-1}: j_{n}\left[\Gamma_{H}, n\right]=j_{n}[\Gamma, n]^{H} \rightarrow \mathcal{L}_{A}\left(n T, n T^{\mathrm{sing}}\right)^{H} .
$$

The global meromorphic sections of the logarithmic-canonical line bundle $\Omega_{A^{\prime}}^{2}\left(T^{\prime}\right)$ are in a bijective correspondence with the families $\left(f_{\alpha}, U_{\alpha}\right)_{\alpha \in S}$ of local meromorphic defining equations $f_{\alpha}: U_{\alpha} \rightarrow \mathbb{C}$ of the logarithmic-canonical divisor $L+T^{\prime}$. We construct local meromorphic $G$-invariant equations $g_{\alpha}: V_{\alpha} \rightarrow \mathbb{C}$ of $T$ and pull-back to $\left(f_{\alpha}=\xi^{*} g_{\alpha}, U_{\alpha}=\xi^{-1}\left(V_{\alpha}\right)\right)_{\alpha \in S}$. Let $F_{A}: \widetilde{A}=\mathbb{C}^{2} \rightarrow A$ be the universal covering map of $A$. Then for any point $p \in A$ choose a lifting $\widetilde{p} \in F_{A}^{-1}(p)$ and a sufficiently small neighborhood $\widetilde{W}$ of $\widetilde{p}$ on $\widetilde{A}$, which is contained in the interior of a $\pi_{1}(A)$-fundamental domain on $\widetilde{A}$, centered at $\widetilde{p}$. The $G$-invariant open neighborhood $W=\cap_{g \in G} g \widetilde{W}$ of $\widetilde{p}$ on $\widetilde{A}$ intersects $F_{A}^{-1}(T)$ in lines with local equations $l_{j}(u, v)=a_{j}(\widetilde{p}) u+b_{j}(\widetilde{p}) v+c_{j}(\widetilde{p})=0$. The holomorphic function $g(u, v)=\prod_{g \in G} \prod_{j}\left(l_{j}(u, v)\right)$ on $W$ is $G$-invariant and can be viewed as a $G$-invariant local defining equation of $T$ on $V=F_{A}(W)$. Note that $F_{A}$ is locally biholomorphic, so that $V \subset A$ is an open subset, after an eventual shrinking of $\widetilde{W}$. The family $(g, V)_{p \in A}$ of local $G$-invariant defining equations of $T$ pulls-back to a family $\left(f=\xi^{*} g, U=\xi^{-1}(V)\right)_{p \in A}$ of local $G$-invariant sections of $\Omega_{A}^{2}\left(T^{\prime}\right)$. 
ii) Towards the coincidence $\left.\left.\Psi_{n}^{H}\right|_{[(A \backslash T) / H]} \equiv \Phi_{n}^{H}\right|_{\left[\left(\mathbb{B} / \Gamma_{H}\right) \backslash(L / H)\right]}$, let us fix a basis $\left\{\omega_{i} ; 1 \leq i \leq d\right\}$ of $j_{n}\left[\Gamma_{H}, n\right]$ and apply $\left.\mathrm{i}\right)$, in order to conclude that the set $\left\{f_{i}=\operatorname{Trans}_{n}^{H} j_{n}^{-1}\left(\omega_{i}\right) ; 1 \leq i \leq d\right\}$ is a basis of $\mathcal{L}_{A}\left(n T, n T^{\text {sing }}\right)^{H}$. Tensoring by $s^{\otimes(-n)}$ does not alter the ratios $\frac{\omega_{i}}{\omega_{j}}$. The isomorphism $\xi: \operatorname{Mer}(A) \rightarrow \operatorname{Mer}\left(A^{\prime}\right)$ is identical on $(A \backslash T) / H$.

\section{Preliminaries}

In order to specify $A_{-1}^{\prime}=\left(\mathbb{B} / \Gamma_{-1}^{(6,8)}\right)^{\prime}$ let us note that the blow-down $\xi: A_{-1}^{\prime} \rightarrow$ $A_{-1}$ of the (-1)-curves maps $T^{\prime}$ to a divisor $T=\xi\left(T^{\prime}\right)$ with smooth elliptic irreducible components $T_{i}$. Such $T$ are called multi-elliptic divisors. Any irreducible component $T_{i}$ of $T$ lifts to a $\pi_{1}\left(A_{-1}\right)$-orbit of complex lines on the universal cover $\widetilde{A_{-1}^{\prime}}=\mathbb{C}^{2}$. That allows to represent

$$
T_{j}=\left\{(u(\bmod \mathbb{Z}+\mathbb{Z} \mathrm{i}), v(\bmod \mathbb{Z}+\mathbb{Z} \mathrm{i})) ; a_{j} u+b_{j} v+c_{j}=0\right\} .
$$

If $T_{j}$ is defined over the field $\mathbb{Q}(\mathrm{i})$ of Gauss numbers, there is no loss of generality in assuming $a_{j}, b_{j} \in \mathbb{Z}[\mathrm{i}]$ to be Gaussian integers.

Theorem 3 (Holzapfel [4]). Let $A_{-1}=E_{-1} \times E_{-1}$ be the Cartesian square of the elliptic curve $E_{-1}=\mathbb{C} / \mathbb{Z}+\mathbb{Z} \mathrm{i}, \omega_{1}=\frac{1}{2}, \omega_{2}=i \omega_{1}, \omega_{3}=\omega_{1}+\omega_{2}$ be half-periods,

$$
Q_{0}=0(\bmod \mathbb{Z}+\mathbb{Z} \mathrm{i}), \quad Q_{1}=\omega_{1}(\bmod \mathbb{Z}+\mathbb{Z} \mathrm{i}), \quad Q_{2}=\mathrm{i} Q_{1}, \quad Q_{3}=Q_{1}+Q_{2}
$$

be the two-torsion points on $E_{-1}, Q_{i j}=\left(Q_{i}, Q_{j}\right) \in A_{-1}^{2-\text { tor }}$ and

$$
\begin{gathered}
T_{k}=\left\{\left(u(\bmod \mathbb{Z}+\mathbb{Z} \mathrm{i}), v(\bmod \mathbb{Z}+\mathbb{Z} \mathrm{i}) ; u-i^{k} v=0\right\} \quad \text { with } 1 \leq k \leq 4,\right. \\
T_{4+m}=\left\{u(\bmod \mathbb{Z}+\mathbb{Z} \mathrm{i}), v(\bmod \mathbb{Z}+\mathbb{Z} \mathrm{i}) ; u-\omega_{m}=0\right\} \text { for } 1 \leq m \leq 2 \text { and } \\
T_{6+m}=\left\{u(\bmod \mathbb{Z}+\mathbb{Z} \mathrm{i}), v(\bmod \mathbb{Z}+\mathbb{Z} \mathrm{i}) ; v-\omega_{m}=0\right\} \text { for } 1 \leq m \leq 2 .
\end{gathered}
$$

Then the blow-up of $A_{-1}$ at the singular locus $\left(T_{-1}^{(6,8)}\right)^{\operatorname{sing}}=Q_{00}+Q_{33}+$ $\sum_{i=1}^{2} \sum_{j=1}^{2} Q_{i j}$ of the multi-elliptic divisor $T_{-1}^{(6,8)}=\sum_{i=1}^{8} T_{i}$ is a neat toroidal ball quotient compactification $A_{-1}^{\prime}=\left(\mathbb{B} / \Gamma_{-1}^{(6,8)}\right)^{\prime}$.

Theorem 4 (Kasparian and Kotzev [6]). The group $G_{-1}=\operatorname{Aut}\left(A_{-1}, T_{-1}^{(6,8)}\right)=$ $\operatorname{Aut}\left(A_{-1}^{\prime}, T^{\prime}\right)$ of order 64 is generated by the translation $\tau_{33}$ with $Q_{33}$, the multiplications

$$
I=\left(\begin{array}{ll}
\mathrm{i} & 0 \\
0 & 1
\end{array}\right), \quad \text { respectively, } \quad J=\left(\begin{array}{ll}
1 & 0 \\
0 & \mathrm{i}
\end{array}\right)
$$


with $\mathrm{i} \in \mathbb{Z}[\mathrm{i}]$ on the first, respectively, the second factor $E_{-1}$ of $A_{-1}$ and the transposition

$$
\theta=\left(\begin{array}{ll}
0 & 1 \\
1 & 0
\end{array}\right)
$$

of these factors.

Throughout, we use the notations from Theorem 3 and Theorem 4, without mentioning this explicitly. With a slight abuse of notation, we speak of KodairaEnriques classification type, irregularity and geometric genus of $A_{-1} / H, H \leq$ $G_{-1}$, referring actually to a smooth minimal model $Y$ of $A_{-1} / H$.

Theorem 5 (Kasparian and Nikolova [7]). Let

$$
\mathcal{L}: G_{-1} \rightarrow \mathrm{GL}_{2}(\mathbb{Z}[\mathrm{i}])=\left\{g \in \mathbb{Z}[\mathrm{i}]_{2 \times 2} ; \operatorname{det}(g) \in \mathbb{Z}[\mathrm{i}]^{*}=\langle\mathrm{i}\rangle\right\}
$$

be the homomorphism, associating to $g \in G_{-1}$ its linear part $\mathcal{L}$ and

$$
\begin{gathered}
L_{1}\left(G_{-1}\right)=\left\{g \in G_{-1} ; \operatorname{rk}\left(\mathcal{L}(g)-I_{2}\right)=1\right\} \\
=\left\{\tau_{33}^{n} I^{k}, \tau_{33}^{n} J^{k}, \tau_{33}^{n} I^{l} J^{-l} \theta ; 0 \leq n \leq 1, \quad 1 \leq k \leq 3,0 \leq l \leq 3\right\} .
\end{gathered}
$$

Then:

i) $A_{-1} / H$ is an abelian surface for $H=\left\langle\tau_{33}\right\rangle$

ii) $A_{-1} / H$ is a hyperelliptic surface for $H=\left\langle\tau_{33} I^{2}\right\rangle$ or $H=\left\langle\tau_{33} J^{2}\right\rangle$

iii) $A_{-1} / H$ is a ruled surface with an elliptic base for

$H=\langle h\rangle, h \in L_{1}\left(G_{-1}\right) \backslash\left\{\tau_{33} I^{2}, \tau_{33} J^{2}\right\} \quad$ or $H=\left\langle\tau_{33}, h_{o}\right\rangle, h_{o} \in \mathcal{L}\left(L_{1}\left(G_{-1}\right)\right)$

iv) $A_{-1} / H$ is a $\mathrm{K} 3$ surface for $\left\langle\tau_{33}^{n}\right\rangle \neq H \leq K=\operatorname{kerdet} \mathcal{L}$, where

$$
K=\left\{\tau_{33}^{n} I^{k} J^{-k}, \tau_{33}^{n} I^{k} J^{2-k} \theta ; 0 \leq n \leq 1,0 \leq k \leq 3\right\}
$$

v) $A_{-1} / H$ is an Enriques surface for $H=\left\langle I^{2} J^{2}, \tau_{33} I^{2}\right\rangle$

vi) $A_{-1} / H$ is a rational surface for

$$
\begin{gathered}
\langle h\rangle \leq H, \quad h \in\left\{\tau_{33}^{n} I J, \tau_{33}^{n} I^{2} J, \tau_{33}^{n} I J^{2} ; 0 \leq n \leq 1\right\} \quad \text { or }\left\langle\tau_{33}^{n} I^{2} J^{2}, h_{1}\right\rangle \leq H, \\
h_{1} \in\left\{I^{2 m} J^{2-2 m}, \tau_{33}^{m} I, \tau_{33}^{m} J, \tau_{33}^{m} I^{l} J^{-l} \theta ; 0 \leq m \leq 1,0 \leq l \leq 3\right\}, 0 \leq n \leq 1 .
\end{gathered}
$$

The following lemma specifies some known properties of Weierstrass $\sigma$-function over Gaussian integers $\mathbb{Z}[\mathrm{i}]$. 
Lemma 6. Let $\sigma(z)=z \prod_{\lambda \in \mathbb{Z}[\mathrm{i}] \backslash\{0\}}\left(1-\frac{z}{\lambda}\right)^{\frac{z}{\lambda}+\frac{1}{2}\left(\frac{z}{\lambda}\right)^{2}}$ be the Weierstrass $\sigma$-function, associated with the lattice $\mathbb{Z}[\mathrm{i}]$ of $\mathbb{C}$. Then:

i) $\sigma\left(\mathrm{i}^{k} z\right)=\mathrm{i}^{k} \sigma(z), \quad z \in \mathbb{C}, \quad 0 \leq k \leq 3$

ii) $\frac{\sigma(z+\lambda)}{\sigma(z)}=\varepsilon(\lambda) e^{-\pi \bar{\lambda} z-\frac{\pi}{2}|\lambda|^{2}}, \quad z \in \mathbb{C}, \quad \lambda \in \mathbb{Z}[\mathrm{i}]$, where

$$
\varepsilon(\lambda)=\left\{\begin{aligned}
-1 & \text { if } \lambda \in \mathbb{Z}[\mathrm{i}] \backslash 2 \mathbb{Z}[\mathrm{i}] \\
1 & \text { if } \lambda \in 2 \mathbb{Z}[\mathrm{i}]
\end{aligned}\right.
$$

Proof: i) follows from

$$
\prod_{\lambda \in \mathbb{Z}[\mathrm{i}] \backslash\{0\}}\left(1-\frac{\mathrm{i}^{k} z}{\lambda}\right)^{\frac{\mathrm{i}^{k} z}{\lambda}+\frac{1}{2}\left(\frac{\mathrm{i}^{k} z}{\lambda}\right)^{2}}=\prod_{\mu=\frac{\lambda}{\mathrm{i} k} \in \mathbb{Z}[\mathrm{i}] \backslash\{0\}}\left(1-\frac{z}{\mu}\right)^{\frac{z}{\mu}+\frac{1}{2}\left(\frac{z}{\mu}\right)^{2}} .
$$

ii) According to Lang's book [8]

$$
\frac{\sigma(z+\lambda)}{\sigma(z)}=\varepsilon(\lambda) \mathrm{e}^{\eta(\lambda)\left(z+\frac{\lambda}{2}\right)}, \quad z \in \mathbb{C}, \quad \lambda \in \mathbb{Z}[\mathrm{i}]
$$

where $\eta: \mathbb{Z}[\mathrm{i}] \rightarrow \mathbb{C}$ is the homomorphism of $\mathbb{Z}$-modules, related to Weierstrass $\zeta$-function $\zeta(z)=\frac{\sigma^{\prime}(z)}{\sigma(z)}$ by the identity $\zeta(z+\lambda)=\zeta(z)+\eta(\lambda)$. It suffices to establish that $\eta(\lambda)=-\pi \bar{\lambda}, \lambda \in \mathbb{Z}[\mathrm{i}]$. Recall from [8] Legendre's equality $\eta(\mathrm{i})-\mathrm{i} \eta(1)=2 \pi \mathrm{i}$, in order to derive

$$
\eta(\lambda)=\frac{\lambda+\bar{\lambda}}{2} \eta(1)+\frac{\lambda-\bar{\lambda}}{2 \mathrm{i}} \eta(\mathrm{i})=(\eta(1)+\pi) \lambda-\pi \bar{\lambda}, \quad \lambda \in \mathbb{Z}[\mathrm{i}] .
$$

Combining with homogeneity $\eta(\mathrm{i} \lambda)=\frac{1}{\mathrm{i}} \eta(\lambda), \lambda \in \mathbb{Z}[\mathrm{i}]$ (cf. [8]), one obtains

$$
(\eta(1)+\pi) \mathrm{i} \lambda+\pi \mathrm{i} \bar{\lambda}=\eta(\mathrm{i} \lambda)=-\mathrm{i} \eta(\lambda)=-(\eta(1)+\pi) \mathrm{i} \lambda+\pi \mathrm{i} \bar{\lambda}, \quad \lambda \in \mathbb{Z}[\mathrm{i}] .
$$

Therefore $\eta(1)=-\pi$ and $\eta(\lambda)=-\pi \bar{\lambda}, \lambda \in \mathbb{Z}[\mathrm{i}]$.

\section{Corollary 7.}

$$
\begin{gathered}
\frac{\sigma\left(z+\omega_{m}\right)}{\sigma\left(z-\omega_{m}\right)}=-\mathrm{e}^{2(-1)^{m} \omega_{m} \pi z} \\
\frac{\sigma\left(z+\omega_{m}+2 \varepsilon \omega_{3-m}\right)}{\sigma\left(z-\omega_{m}\right)}=(-1)^{m+1} \varepsilon \mathrm{ie}^{-\frac{\pi}{2}+2(-1)^{m+1} \varepsilon \omega_{3-m} \pi z+2(-1)^{m} \omega_{m} \pi z} \\
\frac{\sigma\left(z-\omega_{m}+2 \varepsilon \omega_{3-m}\right)}{\sigma\left(z-\omega_{m}\right)}=(-1)^{m+1} \varepsilon \mathrm{ie}^{-\frac{\pi}{2}+2(-1)^{m+1} \varepsilon \omega_{3-m} \pi z} .
\end{gathered}
$$

for the half-periods $\omega_{1}=\frac{1}{2}, \omega_{2}=\mathrm{i} \omega_{1}$ and $\varepsilon= \pm 1$. 


\section{Corollary 8.}

$$
\begin{gathered}
\frac{\sigma\left(z+2 \varepsilon \omega_{m}\right)}{\sigma(z-1)}=\mathrm{e}^{-\pi z+(-1)^{m} 2 \varepsilon \pi \omega_{m} z} \\
\frac{\sigma\left(z+(-1)^{m} \omega_{m}+\varepsilon(-1)^{m} \omega_{3-m}\right)}{\sigma\left(z-(-1)^{m} \omega_{m}+(-1)^{m} \omega_{3-m}\right)}=-\mathrm{i}^{(-1)^{m} \frac{(1+\varepsilon)}{2}} \mathrm{e}^{2 \omega_{m} \pi z+(1-\varepsilon) \omega_{3-m} \pi z .}
\end{gathered}
$$

for the half-periods $\omega_{1}=\frac{1}{2}, \omega_{2}=\mathrm{i} \omega_{1}$ and $\varepsilon= \pm 1$.

Corollary 7 and Corollary 8 follow from Lemma 6 ii) and $\bar{\omega}_{m}=(-1)^{m+1} \omega_{m}$, $\omega_{m}^{2}=\frac{(-1)^{m+1}}{4}$.

In [5] the map $\Phi: \widetilde{\mathbb{B} / \Gamma_{-1}^{(6,8)}} \rightarrow \mathbb{P}\left(\left[\Gamma_{-1}^{(6,8)}, 1\right]\right)$ is shown to be a regular embedding. This is done by constructing a $\mathbb{C}$-basis of $\mathcal{L}=\mathcal{L}_{A_{-1}}\left(T_{-1}^{(6,8)},\left(T_{-1}^{(6,8)}\right)^{\text {sing }}\right)$, consisting of binary parallel or triangular $\sigma$-quotients. An abelian function $f_{\alpha, \beta} \in \mathcal{L}$ is binary parallel if the pole divisor $\left(f_{\alpha, \beta}\right)_{\infty}=T_{\alpha}+T_{\beta}$ consists of two disjoint smooth elliptic curves $T_{\alpha}$ and $T_{\beta}$. A $\sigma$-quotient $f_{i, \alpha, \beta} \in \mathcal{L}$ is triangular if $T_{i} \cap T_{\alpha} \cap T_{\beta}=\emptyset$ and any two of $T_{i}, T_{\alpha}$ and $T_{\beta}$ intersect in a single point.

\section{Theorem 9 (Kasparian and Kotzev [5]). Let}

$$
\begin{gathered}
\Sigma_{12}(z)=\frac{\sigma(z-1) \sigma\left(z+\omega_{1}-\omega_{2}\right)}{\sigma\left(z-\omega_{1}\right) \sigma\left(z-\omega_{2}\right)}, \quad \Sigma_{1}=\frac{\sigma\left(u-\mathrm{i} v+\omega_{3}\right)}{\sigma(u-\mathrm{i} v)} \\
\Sigma_{2}=\frac{\sigma\left(u+v+\omega_{3}\right)}{\sigma(u+v)}, \quad \Sigma_{3}=\frac{\sigma\left(u+\mathrm{i} v+\omega_{3}\right)}{\sigma(u+\mathrm{i} v)}, \quad \Sigma_{4}=\frac{\sigma\left(u-v+\omega_{3}\right)}{\sigma(u-v)} \\
\Sigma_{5}=\frac{\sigma\left(u-\omega_{2}\right)}{\sigma\left(u-\omega_{1}\right)}, \quad \Sigma_{6}=\frac{\sigma\left(u-\omega_{1}\right)}{\sigma\left(u-\omega_{2}\right)}, \quad \Sigma_{7}=\frac{\sigma\left(v-\omega_{2}\right)}{\sigma\left(v-\omega_{1}\right)}, \quad \Sigma_{8}=\frac{\sigma\left(v-\omega_{1}\right)}{\sigma\left(v-\omega_{2}\right)} .
\end{gathered}
$$

Then:

i) the space $\mathcal{L}=\mathcal{L}_{A_{-1}}\left(T_{\sqrt{-1}}^{(6,8)},\left(T_{\sqrt{-1}}^{(6,8)}\right)^{\text {sing }}\right)$ contains the binary parallel $\sigma$-quotients $f_{56}(u, v)=\Sigma_{12}(u), f_{78}(u, v)=\Sigma_{12}(v)$ and the triangular $\sigma$-quotients

$$
\begin{array}{lll}
f_{157}=\mathrm{ie}^{-\frac{\pi}{2}+\pi u} \Sigma_{1} \Sigma_{5} \Sigma_{7}, & f_{168}=-\mathrm{e}^{-\pi-\pi \mathrm{i} u-\pi v-\pi \mathrm{i} v} \Sigma_{1} \Sigma_{6} \Sigma_{8} \\
f_{357}=-\mathrm{e}^{-\pi+\pi u+\pi v+\pi \mathrm{i} v} \Sigma_{3} \Sigma_{5} \Sigma_{7}, & f_{368}=-\mathrm{ie}^{-\frac{\pi}{2}-\pi \mathrm{i} u} \Sigma_{3} \Sigma_{6} \Sigma_{8} \\
f_{258}=\mathrm{e}^{-\pi+\pi u-\pi \mathrm{i} v} \Sigma_{2} \Sigma_{5} \Sigma_{8}, & f_{267}=\mathrm{e}^{-\pi-\pi \mathrm{i} u+\pi v} \Sigma_{2} \Sigma_{6} \Sigma_{7} \\
f_{458}=-\mathrm{ie}^{-\frac{\pi}{2}+\pi u-\pi v} \Sigma_{4} \Sigma_{5} \Sigma_{8}, & f_{467}=\mathrm{ie}^{-\frac{\pi}{2}-\pi \mathrm{i} u+\pi \mathrm{i} v} \Sigma_{4} \Sigma_{6} \Sigma_{7}
\end{array}
$$

ii) $a \mathbb{C}$-basis of $\mathcal{L}$ is

$$
f_{o}:=1, f_{1}:=f_{157}, f_{2}:=f_{258}, f_{3}:=f_{368}, f_{4}:=f_{467}, f_{5}:=f_{56}, f_{6}:=f_{78} \text {. }
$$




\section{Technical Preparation}

The group $G_{-1}=\operatorname{Aut}\left(A_{-1}, T_{-1}^{(6,8)}\right)$ permutes the eight irreducible components of $T_{-1}^{(6,8)}$ and the $\Gamma_{-1}^{(6,8)}$-cusps. For any subgroup $H$ of $G_{-1}$, the $\Gamma_{H}$-cusps are the $H$-orbits of $\partial_{\Gamma_{-1}^{(6,8)}} \mathbb{B} / \Gamma_{-1}^{(6,8)}=\left\{\kappa_{i} ; 1 \leq i \leq 8\right\}$.

Lemma 10. If $\varphi: G_{-1} \rightarrow S_{8}\left(\kappa_{1}, \ldots, \kappa_{8}\right)$ is the natural representation of $G_{-1}=$ Aut $\left(A_{-1}, T_{-1}^{(6,8)}\right)$ in the symmetric group of the $\Gamma_{-1}^{(6,8)}$-cusps, then

$$
\begin{aligned}
\varphi\left(\tau_{33}\right) & =\left(\kappa_{5}, \kappa_{6}\right)\left(\kappa_{7}, \kappa_{8}\right), & \varphi(I) & =\left(\kappa_{1}, \kappa_{4}, \kappa_{3}, \kappa_{2}\right)\left(\kappa_{5}, \kappa_{6}\right) \\
\varphi(J) & =\left(\kappa_{1}, \kappa_{2}, \kappa_{3}, \kappa_{4}\right)\left(\kappa_{7}, \kappa_{8}\right), & & \varphi(\theta)=\left(\kappa_{1}, \kappa_{3}\right)\left(\kappa_{5}, \kappa_{7}\right)\left(\kappa_{6}, \kappa_{8}\right) .
\end{aligned}
$$

Proof: The $\Gamma_{-1}^{(6,8)}$-cusps $\kappa_{i}$ are obtained by contraction of the proper transforms $T_{i}^{\prime}$ of $T_{i}$ under the blow-up of $A_{-1}$ at $\left(T_{-1}^{(6,8)}\right)^{\operatorname{sing}}$. Therefore the representations of $G_{-1}$ in the permutation groups of $\left\{T_{i} ; 1 \leq i \leq 8\right\},\left\{T_{i}^{\prime} ; 1 \leq i \leq 8\right\}$ and $\left\{\kappa_{i} ; 1 \leq i \leq 8\right\}$ coincide.

According to $\tau_{33}\left(u-\mathrm{i}^{k} v\right)=u-\mathrm{i}^{k} v+\left(1-\mathrm{i}^{k}\right) \omega_{3}=u-\mathrm{i}^{k} v(\bmod \mathbb{Z}+\mathbb{Z} \mathrm{i})$, the translation $\tau_{33}$ acts identically on $T_{1}, T_{2}, T_{3}, T_{4}$. Further, $\tau_{33}\left(u-\omega_{m}\right)=$ $u+\omega_{3-m} \equiv u-\omega_{3-m}(\bmod \mathbb{Z}+\mathbb{Z} \mathrm{i})$ reveals the permutation $\left(T_{5}, T_{6}\right)\left(T_{7}, T_{8}\right)$ of the last four components of $T_{-1}^{(6,8)}$.

Due to the identity $I\left(u-\mathrm{i}^{k} v\right)=\mathrm{i} u-\mathrm{i}^{k} v=\mathrm{i}\left(u-\mathrm{i}^{k-1} v\right)$, the automorphism $I$ induces the permutation $\left(T_{1}, T_{4}, T_{3}, T_{2}\right)$ of the first four components of $T_{-1}^{(6,8)}$. Further, $I\left(u-\omega_{m}\right)=\mathrm{i}\left(u \pm \omega_{3-m}\right)$ reveals that $I$ permutes $T_{5}$ with $T_{6}$. Note that $I$ acts identically on $v$ and fixes $T_{7}, T_{8}$.

In a similar vein, $J\left(u-\mathrm{i}^{k} v\right)=u-\mathrm{i}^{k+1} v, J\left(v-\omega_{m}\right)=\mathrm{i}\left(v \pm \mathrm{i} \omega_{3-m}\right)$ determine that $\varphi(J)=\left(\kappa_{1}, \kappa_{2}, \kappa_{3}, \kappa_{4}\right)\left(\kappa_{7}, \kappa_{8}\right)$. According to $\theta\left(u-\mathrm{i}^{k} v\right)=v-$ $\mathrm{i}^{k} u=-\mathrm{i}^{k}\left(u-\mathrm{i}^{-k} v\right)$ and $\theta\left(u-\omega_{m}\right)=v-\omega_{m}$, one concludes that $\varphi(\theta)=$ $\left(\kappa_{1}, \kappa_{3}\right)\left(\kappa_{5}, \kappa_{7}\right)\left(\kappa_{6}, \kappa_{8}\right)$.

The following lemma incorporates several arguments, which will be applied repeatedly towards determination of the target $\mathbb{P}\left(\left[\Gamma_{H}, 1\right]\right)$ and the rank of the logarithmic canonical map $\Phi^{H}$.

Lemma 11. In the notations from Theorem 9, for an arbitrary subgroup $H$ of $G_{-1}=\operatorname{Aut}\left(A_{-1}, T_{-1}^{(6,8)}\right)$ and any $f \in \mathcal{L}=\mathcal{L}_{A_{-1}}\left(T_{-1}^{(6,8)},\left(T_{-1}^{(6,8)}\right)^{\text {sing }}\right)$, let $R_{H}(f)=\sum_{h \in H} h(f)$ be the value of Reynolds operator $R_{H}$ of $H$ on $f$. 
i) The space $\mathcal{L}^{H}$ of the $H$-invariants of $\mathcal{L}$ is spanned by $\left\{R_{H}\left(f_{i}\right) ; 0 \leq i \leq 6\right\}$.

ii) Let $T_{i} \subset\left(R_{H}\left(f_{i, \alpha_{1}, \beta_{1}}\right)\right)_{\infty},\left(R_{H}\left(f_{i, \alpha_{2}, \beta_{2}}\right)\right)_{\infty} \subseteq \operatorname{Orb}_{H}\left(T_{i}\right)+\sum_{\alpha=5}^{8} T_{\alpha}$ for some $1 \leq i \leq 4,5 \leq \alpha_{j} \leq 6,7 \leq \beta_{j} \leq 8$. Then

$$
R_{H}\left(f_{i, \alpha_{2}, \beta_{2}}\right) \in \operatorname{Span}_{\mathbb{C}}\left(1, R_{H}\left(f_{56}\right), R_{H}\left(f_{78}\right), R_{H}\left(f_{i, \alpha_{1}, \beta_{1}}\right)\right) .
$$

iii) Let $\bar{\kappa}_{i_{1}}, \ldots, \bar{\kappa}_{i_{p}}$ with $1 \leq i_{1}<\ldots<i_{p} \leq 4$ be different $\Gamma_{H^{-}}$cusps

$$
T_{i_{j}} \subset\left(R_{H}\left(f_{i_{j}}\right)\right)_{\infty} \subseteq \operatorname{Orb}_{H}\left(T_{i_{j}}\right)+\sum_{\alpha=5}^{8} T_{\alpha} \quad \text { for all } \quad 1 \leq j \leq p
$$

and $B$ be a $\mathbb{C}$-basis of $\mathcal{L}_{2}^{H}=\mathcal{L}_{A_{-1}}\left(\sum_{\alpha=5}^{8} T_{\alpha}\right)^{H}$. Then the set

$$
\left\{R_{H}\left(f_{i_{j}, \alpha_{j}, \beta_{j}}\right) ; 1 \leq j \leq p\right\} \cup B
$$

consists of linearly independent invariants over $\mathbb{C}$.

iv) If $R_{j}=R_{H}\left(f_{j, \alpha_{j}, \beta_{j}}\right) \not \equiv$ const, $\left.R_{j}\right|_{T_{j}}=\infty$ and $R_{i}=R_{H}\left(f_{i, \alpha_{i}, \beta_{i}}\right)$ has $\left.R_{i}\right|_{T_{j}} \not \equiv$ const then for any subgroup $H_{o}$ of $H$ the projective maps

$$
\Psi^{H_{o}}: X / H_{o} \cdots \mathbb{P}\left(\mathcal{L}^{H_{o}}\right), \quad \Phi^{H_{o}}: \widehat{\mathbb{B} / \Gamma_{H_{o}}} \cdots \mathbb{P}\left(j_{1}\left[\Gamma_{H_{o}}, 1\right]\right)
$$

are of $\operatorname{rank} \operatorname{rk} \Phi^{H_{o}}=\operatorname{rk} \Psi^{H_{o}}=2$.

v) If the group $H^{\prime}$ is obtained from the group $H$ by replacing all $\tau_{33}^{n} I^{k} J^{l} \theta^{m} \in$ $H$ with $\tau_{33}^{n} I^{l} J^{k} \theta^{m}$, then the spaces of modular forms $j_{1}\left[\Gamma_{H^{\prime}}, 1\right] \simeq j_{1}\left[\Gamma_{H}, 1\right]$ are isomorphic and the logarithmic-canonical maps have equal rank $\mathrm{rk} \Phi^{H}=$ $\operatorname{rk} \Phi^{H^{\prime}}$.

Proof: i) By Theorem 9 ii), $\mathcal{L}=\operatorname{Span}_{\mathbb{C}}\left(f_{i} ; 0 \leq 6\right)$. Therefore any $f \in \mathcal{L}$ is a $\mathbb{C}$-linear combination $f=\sum_{i=0}^{6} c_{i} f_{i}$. Due to $H$-invariance of $f$ and the linearity of the representation of $H$ in $\operatorname{Aut}(\mathcal{L})$, Reynolds operator

$$
|H| f=R_{H}(f)=\sum_{i=0}^{6} c_{i} R_{H}\left(f_{i}\right) .
$$

ii) Let $\omega_{s} \in j_{1}\left[\Gamma_{-1}^{(6,8)}, 1\right]^{H}$ are the modular forms, which transfer to $R_{H}\left(f_{i, \alpha_{s}, \beta_{s}}\right)$, $1 \leq s \leq 2$. Since $\omega_{1}\left(\kappa_{i}\right) \neq 0$, there exists $c_{i} \in \mathbb{C}$, such that $\omega_{i}^{\prime}=\omega_{2}-c_{i} \omega_{1}$ vanishes at $\kappa_{i}$. By the assumption $\left(R_{H}\left(f_{i, \alpha_{s}, \beta_{s}}\right)\right)_{\infty} \subseteq \operatorname{Orb}_{H}\left(T_{i}\right)+\sum_{\alpha=5}^{8} T_{\alpha}$, the transfer $F_{i} \in \mathcal{L}^{H}$ of $\omega_{i}^{\prime}$ belongs to $\operatorname{Span}_{\mathbb{C}}\left(1, f_{56}, f_{78}\right)^{H}=\operatorname{Span}_{\mathbb{C}}\left(1, R_{H}\left(f_{56}\right), R_{H}\left(f_{78}\right)\right)$. 
iii) As far as the transfer $\operatorname{Trans}_{1}^{H}: j_{1}\left[\Gamma_{H}, 1\right] \rightarrow \mathcal{L}$ is a $\mathbb{C}$-linear isomorphism, it suffices to establish the linear independence of the corresponding modular forms $\left\{\omega_{i_{j}} ; 1 \leq j \leq p\right\} \cup\left\{\omega_{b} ; b \in B\right\}$. Evaluating the $\mathbb{C}$-linear combination $\sum_{j=1}^{p} c_{i_{j}} \omega_{i_{j}}+$ $\sum_{b \in B} c_{b} \omega_{b}=0$ at $\bar{\kappa}_{i_{1}}, \ldots, \bar{\kappa}_{i_{p}}$, one obtains $c_{i_{j}}=0$, according to $\omega_{i_{j}}\left(\bar{\kappa}_{i_{s}}\right)=\delta_{j}^{s}=$ $\left\{\begin{array}{cc}1 & \text { for } j=s \\ 0 & \text { for } j \neq s\end{array}\right.$ and $\omega_{b}\left(\bar{\kappa}_{i_{j}}\right)=0, b \in B, 1 \leq j \leq p$. Then $\sum_{b \in B} \omega_{b}=0$ requires the vanishing of all $c_{b}$, due to the linear independence of $B$.

iv) If $H_{o}$ is a subgroup of $H$ then $\mathcal{L}^{H}$ is a subspace of $\mathcal{L}^{H_{o}}, j_{1}\left[\Gamma_{H}, 1\right]$ is a subspace of $j_{1}\left[\Gamma_{H_{o}}, 1\right]$ and $\Psi^{H}=\operatorname{pr}^{\mathcal{L}} \Psi^{H_{o}}, \Phi^{H}=\operatorname{pr}^{\Gamma_{H}} \Phi^{H_{o}}$ for the projections $\mathrm{pr}^{\mathcal{L}}$ : $\mathbb{P}\left(\mathcal{L}^{H_{o}}\right) \rightarrow \mathbb{P}\left(\mathcal{L}^{H}\right), \operatorname{pr}^{\Gamma_{H}}: \mathbb{P}\left(j_{1}\left[\Gamma_{H_{o}}, 1\right]\right) \rightarrow \mathbb{P}\left(j_{1}\left[\Gamma_{H}, 1\right]\right)$. That is why, it suffices to justify that $\operatorname{rk} \Phi^{H}=\operatorname{rk} \Psi^{H}=2$ is maximal. Assume the opposite and consider $R_{i}, R_{j}: X / H \rightarrow \mathbb{P}^{1}$. The commutative diagram

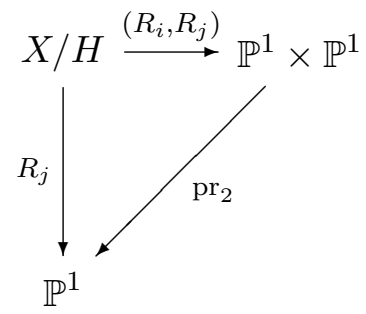

has surjective $R_{j}$, as far as $R_{j} \not \equiv$ const. If the image $C=\left(R_{i}, R_{j}\right)(X / H)$ is a curve, then the projection $\mathrm{pr}_{2}: C \rightarrow \mathbb{P}^{1}$ has only finite fibers. In particular, $\operatorname{pr}_{2}^{-1}(\infty)=R_{i}\left(\left(R_{j}\right)_{\infty}\right) \times \infty \supseteq R_{i}\left(T_{j}\right) \times \infty$ consists of finitely many points. However, $R_{i}\left(T_{j}\right)=\mathbb{P}^{1}$ as an image of the non-constant elliptic function $R_{i}$ : $T_{j} \rightarrow \cdots \mathbb{P}^{1}$. The contradiction implies that $\operatorname{dim}_{\mathbb{C}} C=2$ and $\operatorname{rk} \Psi^{H}=2$.

v) The transposition of the holomorphic coordinates $(u, v) \in \mathbb{C}^{2}$ affects nontrivially the constructed $\sigma$-quotients. However, one can replace the equations $u-\mathrm{i}^{k} v=0$ of $T_{k}, 1 \leq k \leq 4$ by $v-\mathrm{i}^{-k} u=0$ and repeat the above considerations with interchanged $u, v$. The dimension of $j_{1}\left[\Gamma_{H}, 1\right]$ and the rank of $\Phi^{H}$ are invariant under the transposition of the global holomorphic coordinates on $\widetilde{A_{-1}}=\mathbb{C}^{2}$.

With a slight abuse of notation, we write $g(f)$ instead of $g^{*}(f)$, for $g \in G_{-1}$, $f \in \mathcal{L}=\mathcal{L}_{A_{-1}}\left(T_{-1}^{(6,8)},\left(T_{-1}^{(6,8)}\right)^{\text {sing }}\right)$. 
Lemma 12. The generators $\tau_{33}, I, J, \theta$ of $G_{-1}$ act on the binary parallel and triangular $\sigma$-quotients from Corollary 9 as follows:

$$
\begin{aligned}
& 3 \tau_{33}\left(f_{56}\right)=-f_{56}, \\
& \tau_{33}\left(f_{78}\right)=-f_{78} \\
& \tau_{33}\left(f_{157}\right)=-\mathrm{ie}^{\frac{\pi}{2}} f_{168}, \\
& \tau_{33}\left(f_{168}\right)=\mathrm{ie}^{-\frac{\pi}{2}} f_{157}, \\
& \tau_{33}\left(f_{368}\right)=\mathrm{ie}^{\frac{\pi}{2}} f_{357}, \\
& \tau_{33}\left(f_{258}\right)=f_{267}, \\
& \tau_{33}\left(f_{357}\right)=-\mathrm{ie}^{-\frac{\pi}{2}} f_{368} \\
& \tau_{33}\left(f_{458}\right)=-f_{467}, \\
& \tau_{33}\left(f_{467}\right)=-f_{458} \\
& I\left(f_{56}\right)=-\mathrm{i} f_{56}, \\
& I\left(f_{78}\right)=f_{78} \\
& I\left(f_{157}\right)=-\mathrm{i} f_{467}, \\
& I\left(f_{168}\right)=-\mathrm{e}^{-\frac{\pi}{2}} f_{458}, \\
& \tau_{33}\left(f_{267}\right)=f_{258} \\
& I\left(f_{368}\right)=-\mathrm{e}^{\frac{\pi}{2}} f_{258}, \\
& I\left(f_{258}\right)=\mathrm{i} f_{168}, \\
& I\left(f_{357}\right)=\mathrm{i} f_{267} \\
& I\left(f_{458}\right)=-\mathrm{i} f_{368}, \\
& I\left(f_{467}\right)=-\mathrm{e}^{\frac{\pi}{2}} f_{357} \\
& J\left(f_{56}\right)=f_{56}, \\
& J\left(f_{78}\right)=-\mathrm{i} f_{78} \\
& J\left(f_{157}\right)=-\mathrm{ie}^{\frac{\pi}{2}} f_{258}, \\
& J\left(f_{168}\right)=f_{267}, \\
& J\left(f_{357}\right)=\mathrm{ie}^{-\frac{\pi}{2}} f_{458} \\
& J\left(f_{368}\right)=f_{467}, \\
& J\left(f_{258}\right)=f_{357}, \\
& J\left(f_{458}\right)=f_{157}, \\
& J\left(f_{467}\right)=\mathrm{ie}^{\frac{\pi}{2}} f_{168} \\
& \theta\left(f_{56}\right)=f_{78}, \\
& \theta\left(f_{78}\right)=f_{56} \\
& \theta\left(f_{157}\right)=-\mathrm{e}^{\frac{\pi}{2}} f_{357}, \\
& \theta\left(f_{168}\right)=-\mathrm{e}^{-\frac{\pi}{2}} f_{368}, \\
& I\left(f_{267}\right)=-\mathrm{e}^{-\frac{\pi}{2}} f_{157} \\
& \theta\left(f_{368}\right)=-\mathrm{e}^{\frac{\pi}{2}} f_{168}, \\
& \theta\left(f_{258}\right)=f_{267}, \\
& J\left(f_{267}\right)=-\mathrm{ie}^{-\frac{\pi}{2}} f_{368} \\
& \theta\left(f_{458}\right)=f_{467} \text {, } \\
& \theta\left(f_{467}\right)=f_{458} \text {. }
\end{aligned}
$$

Proof: Making use of Lemma 6 and Corollary 8, one computes that

$$
\begin{gathered}
\tau_{33} \sigma(u-1)=-\mathrm{e}^{\pi u+\pi \mathrm{i} u} \sigma\left(u+\omega_{1}-\omega_{2}\right), \quad \tau_{33} \sigma\left(u+\omega_{1}-\omega_{2}\right)=\mathrm{e}^{-2 \pi u} \sigma(u-1) \\
\tau_{33} \sigma\left(u-\omega_{1}\right)=-\mathrm{e}^{\pi \mathrm{i} u} \sigma\left(u-\omega_{2}\right), \quad \tau_{33} \sigma\left(u-\omega_{2}\right)=-\mathrm{e}^{-\pi u} \sigma\left(u-\omega_{1}\right) \\
\tau_{33}\left(\Sigma_{1}\right)=-\mathrm{ie}^{-\frac{\pi}{2}} \Sigma_{1}, \quad \tau_{33}\left(\Sigma_{2}\right)=\mathrm{e}^{-\pi} \Sigma_{2}, \quad \tau_{33}\left(\Sigma_{3}\right)=\mathrm{ie}^{-\frac{\pi}{2}} \Sigma_{3}, \quad \tau_{33}\left(\Sigma_{4}\right)=\Sigma_{4} \\
\tau_{33}\left(\Sigma_{5}\right)=\mathrm{e}^{-\pi u-\pi \mathrm{i} u} \Sigma_{6}, \quad \tau_{33}\left(\Sigma_{6}\right)=\mathrm{e}^{\pi u+\pi \mathrm{i} u} \Sigma_{5} \\
\tau_{33}\left(\Sigma_{7}\right)=\mathrm{e}^{-\pi v-\pi \mathrm{i} v} \Sigma_{8}, \quad \tau_{33}\left(\Sigma_{8}\right)=\mathrm{e}^{\pi v+\pi \mathrm{i} v} \Sigma_{7} \\
I \sigma(u-1)=\mathrm{ie}^{-\pi u+\pi \mathrm{i} u} \sigma(u-1), \quad I \sigma\left(u+\omega_{1}-\omega_{2}\right)=-\mathrm{e}^{\pi u} \sigma\left(u+\omega_{1}-\omega_{2}\right) \\
I \sigma\left(u-\omega_{1}\right)=-\mathrm{ie}^{\pi \mathrm{i} u} \sigma\left(u-\omega_{2}\right), \quad I \sigma\left(u-\omega_{2}\right)=\mathrm{i} \sigma\left(u-\omega_{1}\right) \\
I\left(\Sigma_{1}\right)=\mathrm{ie}^{-\pi \mathrm{i} u+\pi \mathrm{i} v} \Sigma_{4}, \quad I\left(\Sigma_{2}\right)=\mathrm{ie}^{-\pi \mathrm{i} u-\pi v} \Sigma_{1} \\
I\left(\Sigma_{3}\right)=\mathrm{ie}^{-\pi \mathrm{i} u-\pi \mathrm{i} v} \Sigma_{2}, \quad I\left(\Sigma_{4}\right)=\mathrm{ie}^{-\pi \mathrm{i} u+\pi v} \Sigma_{3}
\end{gathered}
$$




$$
\begin{gathered}
I\left(\Sigma_{5}\right)=-\mathrm{e}^{-\pi \mathrm{i} u} \Sigma_{6}, I\left(\Sigma_{6}\right)=-\mathrm{e}^{\pi \mathrm{i} u} \Sigma_{5}, I\left(\Sigma_{7}\right)=\Sigma_{7}, I\left(\Sigma_{8}\right)=\Sigma_{8} \\
J \sigma(v+\mu)=\left.I \sigma(u+\mu)\right|_{u=v}, \quad \mu \in \mathbb{C} \\
4 J\left(\Sigma_{1}\right)=\Sigma_{2}, \quad J\left(\Sigma_{2}\right)=\Sigma_{3}, \quad J\left(\Sigma_{3}\right)=\Sigma_{4}, \quad J\left(\Sigma_{4}\right)=\Sigma_{1} \\
J\left(\Sigma_{5}\right)=\Sigma_{5}, \quad J\left(\Sigma_{6}\right)=\Sigma_{6}, \quad J\left(\Sigma_{7}\right)=-\mathrm{e}^{-\pi \mathrm{i} v} \Sigma_{8}, \quad J\left(\Sigma_{8}\right)=-\mathrm{e}^{\pi \mathrm{i} v} \Sigma_{7} \\
\theta \sigma(u+\mu)=\sigma(v+\mu), \quad \mu \in \mathbb{C} \\
\theta\left(\Sigma_{1}\right)=-\mathrm{ie}^{\pi u+\pi \mathrm{i} v} \Sigma_{3}, \quad \theta\left(\Sigma_{2}\right)=\Sigma_{2} \\
\theta\left(\Sigma_{3}\right)=\mathrm{ie}^{-\pi \mathrm{i} u-\pi v} \Sigma_{1}, \quad \theta\left(\Sigma_{4}\right)=-\mathrm{e}^{\pi u-\pi \mathrm{i} u-\pi v+\pi \mathrm{i} v} \Sigma_{4} \\
\theta\left(\Sigma_{5}\right)=\Sigma_{7}, \theta\left(\Sigma_{6}\right)=\Sigma_{8}, \theta\left(\Sigma_{7}\right)=\Sigma_{5}, \theta\left(\Sigma_{8}\right)=\Sigma_{6} .
\end{gathered}
$$

The following lemma is an immediate consequence of Lemma 6 and Corollary 7.

\section{Lemma 13.}

$$
\begin{gathered}
\left.\frac{f_{157}}{\Sigma_{1}}\right|_{T_{1}}=-\mathrm{ie}^{-\frac{\pi}{2}},\left.\frac{f_{168}}{\Sigma_{1}}\right|_{T_{1}}=\mathrm{e}^{-\pi},\left.\frac{f_{258}}{\Sigma_{2}}\right|_{T_{2}}=\mathrm{e}^{-\pi},\left.\frac{f_{267}}{\Sigma_{2}}\right|_{T_{2}}=\mathrm{e}^{-\pi} \\
\left.\frac{f_{357}}{\Sigma_{3}}\right|_{T_{3}}=\mathrm{e}^{-\pi},\left.\frac{f_{368}}{\Sigma_{3}}\right|_{T_{3}}=\mathrm{ie}^{-\frac{\pi}{2}},\left.\frac{f_{458}}{\Sigma_{4}}\right|_{T_{4}}=-\mathrm{ie}^{-\frac{\pi}{2}},\left.\frac{f_{467}}{\Sigma_{4}}\right|_{T_{4}}=\mathrm{ie}^{-\frac{\pi}{2}} \\
\left.\frac{f_{157}+\mathrm{ie}^{\frac{\pi}{2}} f_{357}}{\Sigma_{5}}\right|_{T_{5}}=0,\left.\frac{f_{258}-\mathrm{ie}^{-\frac{\pi}{2}} f_{458}}{\Sigma_{5}}\right|_{T_{5}}=0 .
\end{gathered}
$$

\section{Lemma 14.}

$$
\begin{gathered}
{\left.\left[\left(f_{157}-\mathrm{ie}^{\frac{\pi}{2}} f_{168}\right)+c\left(f_{357}-\mathrm{ie}^{-\frac{\pi}{2}} f_{368}\right)\right]\right|_{T_{2}}=\mathrm{ie}^{-\frac{\pi}{2}-\pi v}\left(1+c \mathrm{e}^{-\frac{\pi}{2}}\right)} \\
\frac{\sigma\left((1+\mathrm{i}) v+\omega_{3}\right)}{\sigma((1+\mathrm{i}) v)}\left[\mathrm{e}^{(1+\mathrm{i}) \pi v} \frac{\sigma\left(v-\omega_{2}\right)^{2}}{\sigma\left(v-\omega_{1}\right)^{2}}+\mathrm{e}^{-(1+\mathrm{i}) \pi v} \frac{\sigma\left(v-\omega_{1}\right)^{2}}{\sigma\left(v-\omega_{2}\right)^{2}}\right]
\end{gathered}
$$

is non-constant for all $c \in \mathbb{C} \backslash\left\{-\mathrm{e}^{\frac{\pi}{2}}\right\}$.

Proof: Note that

$$
\begin{aligned}
f(v)= & {\left.\left[\left(f_{157}-\mathrm{ie}^{\frac{\pi}{2}} f_{168}\right)+c\left(f_{357}-\mathrm{ie}^{-\frac{\pi}{2}} f_{368}\right)\right]\right|_{T_{2}} } \\
= & {\left[\mathrm{ie}^{-\frac{\pi}{2}-\pi v} \Sigma_{1}(-v, v)-c \mathrm{e}^{-\pi+\pi \mathrm{i} v} \Sigma_{3}(-v, v)\right] } \\
& \times\left[\Sigma_{5}(-v) \Sigma_{7}(v)+\Sigma_{6}(-v) \Sigma_{8}(v)\right] \\
= & \mathrm{i}^{-\frac{\pi}{2}-\pi v}\left(1+c \mathrm{e}^{-\frac{\pi}{2}}\right) \frac{\sigma\left((1+\mathrm{i}) v-\omega_{3}\right)}{\sigma((1+\mathrm{i}) v)} \\
& \times\left[\mathrm{e}^{(1+\mathrm{i}) \pi v} \frac{\sigma\left(v-\omega_{2}\right)^{2}}{\sigma\left(v-\omega_{1}\right)^{2}}+\mathrm{e}^{-(1+\mathrm{i}) \pi v} \frac{\sigma\left(v-\omega_{1}\right)^{2}}{\sigma\left(v-\omega_{2}\right)^{2}}\right]
\end{aligned}
$$


making use of Lemma 6 and Corollary 7. Obviously, $f(v)$ has no poles outside $\mathbb{Q}(\mathrm{i})$. It suffices to justify that $\lim _{v \rightarrow 0} f(v)=\infty$, in order to conclude that $f(v) \not \equiv$ const. To this end, use $\sigma\left(\omega_{2}\right)=\mathrm{i} \sigma\left(\omega_{1}\right)$ to observe that

$$
\left.f(v) \sigma((1+\mathrm{i}) v)\right|_{v=0}=2 \mathrm{ie}^{-\frac{\pi}{2}}\left(1+c \mathrm{e}^{-\frac{\pi}{2}}\right) \sigma\left(\omega_{3}\right) \neq 0
$$

whenever $c \neq-\mathrm{e}^{\frac{\pi}{2}}$, while $\left.\sigma((1+\mathrm{i}) v)\right|_{v=0}=0$.

\section{Basic Results}

Lemma 15. For $H=\left\langle I J^{2}, \tau_{33} J^{2}\right\rangle,\left\langle I^{2} J, \tau_{33} I^{2}\right\rangle$ with rational $A_{-1} / H$ and any $-\mathrm{Id} \in H \leq G_{-1}$, the map $\Phi^{H}: \widehat{\mathbb{B} / \Gamma_{H}} \rightarrow \cdots \mathbb{P}\left(\left[\Gamma_{H}, 1\right]\right)$ is constant.

Proof: By Lemma 11 (iv), the assertion for $\left\langle I^{2} J, \tau_{33} I^{2}\right\rangle$ is a consequence of the one for $\left\langle I J^{2}, \tau_{33} J^{2}\right\rangle$. In the case of $H=\left\langle I J^{2}, \tau_{33} J^{2}\right\rangle$, the space $\mathcal{L}^{H}$ is spanned by Reynolds operators

$$
R_{H}\left(f_{56}\right)=0, \quad R_{H}\left(f_{78}\right)=0
$$

$R_{H}\left(f_{157}\right)=f_{157}+\mathrm{ie}^{\frac{\pi}{2}} f_{168}+\mathrm{e}^{\frac{\pi}{2}} f_{267}-\mathrm{e}^{\frac{\pi}{2}} f_{258}+\mathrm{ie}^{\frac{\pi}{2}} f_{357}-f_{368}+\mathrm{i} f_{467}+\mathrm{i} f_{458}$.

The $\Gamma_{H}$-cusps are $\bar{\kappa}_{1}=\bar{\kappa}_{2}=\bar{\kappa}_{3}=\bar{\kappa}_{4}, \bar{\kappa}_{5}=\bar{\kappa}_{6}$ and $\bar{\kappa}_{7}=\bar{\kappa}_{8}$. By Lemma 13, $\left.\frac{f_{157}+\mathrm{ie}^{\frac{\pi}{2}} f_{168}}{\Sigma_{1}}\right|_{T_{1}}=0$, so that $\left.R_{H}\left(f_{157}\right)\right|_{T_{1}} \neq \infty$. Therefore $R_{H}\left(f_{157}\right) \in \mathcal{L}_{2}^{H}=\mathbb{C}$ and $\operatorname{rk} \Phi^{H}=0$.

It suffices to observe that $-\mathrm{Id}$ changes the signs of the $\mathbb{C}$-basis

$$
f_{56}, f_{78}, f_{157}, f_{258}, f_{368}, f_{467}
$$

of $\mathcal{L}=\mathcal{L}_{A_{-1}}\left(T_{-1}^{(6,8)},\left(T_{-1}^{(6,8)}\right)^{\text {sing }}\right)$. Then for $H_{o}=\langle-\operatorname{Id}\rangle$ the space $\mathcal{L}^{H_{o}}$ is generated by $R_{H_{o}}(1)=1$. Any subgroup $H_{o} \leq H \leq G_{-1}$ decomposes into cosets $H=\cup_{i=1}^{k} h_{i} H_{o}$ and $R_{H}=\sum_{i=1}^{k} h_{i} R_{H_{o}}$ vanishes on (1). Thus, $\mathcal{L}^{H}=\mathbb{C}$ and $\operatorname{rk} \Phi^{H}=0$.

Note that $A_{-1} /\langle-\mathrm{Id}\rangle$ has 16 double points, whose minimal resolution is the Kummer surface $X_{-1}$ of $A_{-1}$. Thus, $H \ni-$ Id exactly when the minimal resolution $Y$ of the singularities of $A_{-1} / H$ is covered by a smooth model of $X_{-1}$. More precisely, all $A_{-1} / H$ with $-\mathrm{Id} \in H$ have vanishing irregularity $0 \leq q\left(A_{-1} / H\right) \leq$ $q\left(X_{-1}\right)=0$. These are the Enriques $A_{-1} /\left\langle-\mathrm{Id}, \tau_{33} I^{2}\right\rangle$, all $\mathrm{K} 3$ quotients $A_{-1} / H$ 
with $\left\langle\tau_{33}^{n}\right\rangle \neq H \leq K=\operatorname{kerdet} \mathcal{L}$, except $A_{-1} /\left\langle\tau_{33}(-\mathrm{Id})\right\rangle$ and the rational $A_{-1} / H$ with $\tau_{33} I J \in H$ for $0 \leq n \leq 1$ or $\left\langle-\mathrm{Id}, h_{1}\right\rangle \leq H$ for

$$
h_{1} \in\left\{I^{2 m} J^{2-2 m}, \quad \tau_{33}^{m} I, \quad \tau_{33}^{m} J, \quad \tau_{33}^{m} I^{l} J^{-l} \theta ; 0 \leq m \leq 1, \quad 0 \leq l \leq 3\right\} .
$$

Lemma 16. The non-trivial subgroups $H \not \supset-\operatorname{Id}$ of $G_{-1}$ are

i) cyclic of order two

$$
H_{2}(m, l)=\left\langle\tau_{33} I^{2 m} J^{2 l}\right\rangle \text { with } 0 \leq m, l \leq 1
$$

$H_{2}^{\theta}(n, k)=\left\langle\tau_{33}^{n} I^{k} J^{-k} \theta\right\rangle$ with $0 \leq n \leq 1,0 \leq k \leq 3, H_{2}^{\prime}=\left\langle I^{2}\right\rangle, H_{2}^{\prime \prime}=\left\langle J^{2}\right\rangle$

ii) cyclic of order four

$$
\begin{aligned}
& H_{4}^{\prime}(n, m)=\left\langle\tau_{33}^{n} I J^{2 m}\right\rangle \text { with } 0 \leq n, m \leq 1 \\
& H_{4}^{\prime \prime}(n, m)=\left\langle\tau_{33}^{n} I^{2 m} J\right\rangle \text { with } 0 \leq n, m \leq 1
\end{aligned}
$$

iii) isomorphic to Klein group $\mathbb{Z}_{2} \times \mathbb{Z}_{2}$

$$
\begin{gathered}
H_{2 \times 2}^{\prime}(m)=\left\langle\tau_{33} J^{2 m}, I^{2}\right\rangle \text { with } 0 \leq m \leq 1 \\
H_{2 \times 2}^{\prime \prime}(m)=\left\langle\tau_{33} I^{2 m}, J^{2}\right\rangle \text { with } 0 \leq m \leq 1 \\
H_{2 \times 2}^{\theta}(k)=\left\langle I^{k} J^{-k} \theta, \tau_{33}\right\rangle \text { with } 0 \leq k \leq 1 \\
H_{2 \times 2}^{\theta}(n, k)=\left\langle\tau_{33}^{n} I^{k} J^{-k} \theta, \tau_{33} I^{2} J^{2}\right\rangle \text { with } 0 \leq n, k \leq 1
\end{gathered}
$$

iv) isomorphic to $\mathbb{Z}_{4} \times \mathbb{Z}_{2}$

$$
\begin{aligned}
& H_{4 \times 2}^{\prime}(m, l)=\left\langle I J^{2 m}, \tau_{33} J^{2 l}\right\rangle \text { with } 0 \leq m, l \leq 1 \\
& H_{4 \times 2}^{\prime \prime}(m, l)=\left\langle I^{2 m} J, \tau_{33} I^{2 l}\right\rangle \text { with } \quad 0 \leq m, l \leq 1 .
\end{aligned}
$$

Proof: If $H$ is a subgroup of $G_{-1}$, which does not contain -Id, then $H \subseteq S=$ $\left\{g \in G_{-1} ;-\right.$ Id $\left.\notin\langle g\rangle\right\}$. Decompose $G_{-1}=G_{-1}^{\prime} \cup G_{-1}^{\prime} \theta$ into cosets modulo the abelian subgroup

$$
G_{-1}^{\prime}=\left\{\tau_{33}^{n} I^{k} J^{l} ; 0 \leq n \leq 1,0 \leq k, l \leq 3\right\} \leq G_{-1} .
$$

The cyclic group, generated by $\left(\tau_{33}^{n} I^{k} J^{l} \theta\right)^{2}=(I J)^{k+l}$ does not contain - Id = $(I J)^{2}$ if and only if $k+l \equiv 0(\bmod 4)$. If $S^{(r)}=\{g \in S ; g$ is of order $r\}$ then

$$
S \cap G_{-1}^{\prime} \theta=\left\{\tau_{33}^{n} I^{k} J^{-k} \theta ; 0 \leq n \leq 1,0 \leq k \leq 3\right\}=S^{(2)} \cap G_{-1}^{\prime} \theta=: S_{1}^{(2)}
$$


and $S \cap G_{-1}^{\prime} \theta \subseteq S^{(2)}$ consists of elements of order two. Concerning $S \cap G_{-1}^{\prime}$, observe that $\left(\tau_{33}^{n} I^{k} J^{k+2 m}\right)^{2}=(I J)^{2 k} \in S$ for $0 \leq n, m \leq 1,0 \leq k \leq 3$ requires $k=2 p$ to be even. Consequently

$$
\begin{aligned}
& \left\{\tau_{33}^{n} I^{k} J^{l} ; k \equiv l(\bmod 2)\right\} \cap S \\
& =\left\{\tau_{33} I^{2 m} J^{2 l}, I^{2}, \quad J^{2} ; 0 \leq m, l \leq 1\right\}=S^{(2)} \cap G_{-1}^{\prime}=: S_{0}^{(2)} \\
& \left\{\tau_{33}^{n} I^{k} J^{l} ; k \equiv l+1(\bmod 2)\right\} \cap S \\
& =\left\{\tau_{33}^{n} I^{2 m+1} J^{2 l}, \tau_{33}^{n} I^{2 m} J^{2 l+1} ; 0 \leq n, m, l \leq 1\right\}=S^{(4)} .
\end{aligned}
$$

In such a way, one obtains $S=\{\operatorname{Id}\} \cup S_{0}^{(2)} \cup S_{1}^{(2)} \cup S^{(4)}$ of cardinality $|S|=31$. If a subgroup $H$ of $G_{-1}$ is contained in $S$, then $|H| \leq|S|=31$ divides $\left|G_{-1}\right|=64$, i.e., $|H|=1,2,4,8$ or 16 . The only subgroup $H<G_{-1}$ of $|H|=1$ is the trivial one $H=\{\operatorname{Id}\}$. The subgroups $-\mathrm{Id} \notin H<G_{-1}$ of order two are the cyclic ones, generated by $h \in S_{0}^{(2)} \cup S_{1}^{(2)}$. We denote $H_{2}(m, l)=\left\langle\tau_{33} I^{2 m} J^{2 l}\right\rangle$ for $0 \leq m, l \leq 1, H_{2}^{\theta}(n, k)=\left\langle\tau_{33}^{n} I^{k} J^{-k} \theta\right\rangle$ for $0 \leq n \leq 1,0 \leq k \leq 3$ and $H_{2}^{\prime}=\left\langle I^{2}\right\rangle, H_{2}^{\prime \prime}=\left\langle J^{2}\right\rangle$.

For any $h \in S^{(4)}$ one has $\langle h\rangle=\left\langle h^{3}\right\rangle$, so that the subgroups - Id $\notin H \simeq \mathbb{Z}_{4}$ of $G_{-1}$ are depleted by $H_{4}^{\prime}(n, m)=\left\langle\tau_{33}^{n} I J^{2 m}\right\rangle, H_{4}^{\prime \prime}(n, m)=\left\langle\tau_{33}^{n} I^{2 m} J\right\rangle$ with $0 \leq n, m \leq 1$.

The subgroups - Id $\notin H \simeq \mathbb{Z}_{2} \times \mathbb{Z}_{2}$ of $G_{-1}$ are generated by commuting $g_{1}, g_{2} \in$ $S^{(2)}=S_{0}^{(2)} \cup S_{1}^{(2)}$. If $g_{1}, g_{2} \in S_{1}^{(2)}$ then $g_{1} g_{2} \in G_{-1}^{\prime}$, so that one can always assume that $g_{2} \in S_{0}^{(2)}$. Any $g_{1} \neq g_{2}$ from $S_{0}^{(2)} \subset G_{-1}^{\prime}$ generate a Klein group of order 4. Moreover, if

$$
S_{0,1}^{(2)}=\left\{\tau_{33} I^{2 m} J^{2 l} ; 0 \leq m, l \leq 1\right\}, \quad S_{0,0}^{(2)}=\left\{I^{2}, J^{2}\right\}
$$

then for any $g_{1}, g_{2} \in S_{0,1}^{(2)}$ with $g_{1} g_{2} \in S$ there follows $g_{1} g_{2} \in S_{0,0}^{(2)}$. Thus, any $S_{0}^{(2)} \supset H \simeq \mathbb{Z}_{2} \times \mathbb{Z}_{2}$ has at least one generator $g_{2} \in S_{0,0}^{(2)}$. The requirement $I^{2} J^{2}=-$ Id $\notin H$ specifies that $g_{1} \in S_{0,1}^{(2)}$. In the case of $g_{2}=I^{2}$ there is no loss of generality to choose $g_{1}=\tau_{33} J^{2 m}$, in order to form $H_{2 \times 2}^{\prime}(m)$. Similarly, for $g_{2}=J^{2}$ it suffices to take $g_{1}=\tau_{33} I^{2 m}$, while constructing $H_{2 \times 2}^{\prime \prime}(m)$. In order to determine the subgroups $-\mathrm{Id} \notin H=\left\langle g_{1}\right\rangle \times\left\langle g_{2}\right\rangle \simeq \mathbb{Z}_{2} \times \mathbb{Z}_{2}$ with $g_{1} \in S_{1}^{(2)}, g_{2} \in$ $S_{0}^{(2)}$, note that $g_{1}=\tau_{33}^{n} I^{k} J^{-k} \theta$ does not commute with $I^{2}, J^{2}$ and commutes with $g_{2}=\tau_{33} I^{2 m} J^{2 l}$ if and only if $2 m \equiv 2 l(\bmod 4)$, i.e., $0 \leq m=l \leq 1$. Bearing in mind that $\left\langle\tau_{33}^{n} I^{k} J^{-k} \theta, \tau_{33} I^{2 m} J^{2 m}\right\rangle=\left\langle\tau_{33}^{n+1} I^{k+2 m} J^{-k+2 m} \theta, \tau_{33} I^{2 m} J^{2 m}\right\rangle$, 
one restricts the values of $k$ to $0 \leq k \leq 1$. For $m=0$ denote $H_{2 \times 2}^{\theta}(k)=$ $\left\langle I^{k} J^{-k} \theta, \tau_{33}\right\rangle$. For $m=1$ put $H_{2 \times 2}^{\theta}(n, k)=\left\langle\tau_{33}^{n} I^{k} J^{-k} \theta, \tau_{33} I^{2} J^{2}\right\rangle$.

Let - Id $\notin H \subset S$ be a subgroup of order 8 . The non-abelian such $H$ are isomorphic to quaternionic group $\mathbb{Q}_{8}=\left\langle s, t ; s^{4}=\mathrm{Id}, s^{2}=t^{2}\right.$, sts $\left.=t\right\rangle$ or to dihedral group $\mathbb{D}_{4}=\left\langle s, t ; s^{4}=\mathrm{Id}, t^{2}=\mathrm{Id}, \quad s t s=t\right\rangle$. Note that $s \in S^{(4)}$ and $s t s=t$ require $s t \neq t s$. As far as $S^{(4)} \cup S_{0}^{(2)} \subset G_{-1}^{\prime}$ for the abelian group $G_{-1}^{\prime}=\left\langle\tau_{33}, I, J\right\rangle$, it suffices to consider $t=\tau_{33}^{n} I^{k} J^{-k} \theta \in S_{1}^{(2)}$ and $s=\tau_{33}^{m} I^{p} J^{2 l+1-p} \in S^{(4)}$ with $0 \leq n, m, l \leq 1,0 \leq p, k \leq 3$. However, $s t s=\tau_{33}^{n} I^{k+2 l+1} J^{k+2 l+1} \theta \neq t$ reveals the non-existence of a non-abelian group -Id $\notin H \leq G_{-1}$ of order 8 .

The abelian groups $H \subset S=\{\operatorname{Id}\} \cup S^{(2)} \cup S^{(4)}$ of order 8 are isomorphic to $\mathbb{Z}_{4} \times \mathbb{Z}_{2}$ or $\mathbb{Z}_{2} \times \mathbb{Z}_{2} \times \mathbb{Z}_{2}$. Any $\mathbb{Z}_{4} \times \mathbb{Z}_{2} \simeq H \subset S$ is generated by $s=$ $\tau_{33}^{m} I^{p} J^{2 l+1-p} \in S^{(4)}$ and $t \in S_{0}^{(2)}$, as far as $t^{\prime}=\tau_{33}^{n} I^{k} J^{-k} \theta \in S_{1}^{(2)}$ has

$$
s t^{\prime}=\tau_{33}^{m+n} I^{p+k} J^{2 l+1-(p+k)} \theta \neq \tau_{33}^{m+n} I^{2 l+1-(p-k)} J^{p-k} \theta=t^{\prime} s .
$$

For $s=\tau_{33}^{n} I^{2 m+1} J^{2 l} \in S^{(4)}$ there holds $\langle s, t\rangle=\left\langle s^{3}, t\right\rangle$ and it suffices to consider $s=\tau_{33}^{n} I J^{2 l}$. Further, $t \notin\left\langle s^{2}\right\rangle=\left\langle I^{2}\right\rangle$ and $s^{2} t \neq-$ Id specify that $t=\tau_{33} I^{2 p} J^{2 q}$ for some $0 \leq p, q \leq 1$. Replacing eventually $t$ by $t s^{2}=t I^{2}$, one attains $t=$ $\tau_{33} J^{2 q}$. On the other hand, the generator $s=\tau_{33} I J^{2 l} \in S^{(4)}$ of $H=\langle s, t\rangle$ can be restored by $s t=I J^{2(l+q)}$, so that $H=H_{4 \times 2}^{\prime}(l, q)=\left\langle I J^{2 l}, \tau_{33} J^{2 q}\right\rangle$ for some $0 \leq l, q \leq 1$. Exchanging $I$ with $J$, one obtains the remaining groups $H_{4 \times 2}^{\prime \prime}(l, q)=\left\langle I^{2 l} J, \tau_{33} I^{2 q}\right\rangle \simeq \mathbb{Z}_{4} \times \mathbb{Z}_{2}$, contained in $S$.

If - Id $\notin H \subset S$ is isomorphic to $\mathbb{Z}_{2} \times \mathbb{Z}_{2} \times \mathbb{Z}_{2}$ then arbitrary different elements $s, t, r \in H$ of order two commute and generate $H$. For any $x \in S$ and $M \subseteq S$, consider the centralizer $C_{M}(x)=\{y \in M ; x y=y x\}$ of $x$ in $M$. Looking for $s \in S^{(2)}, t \in C_{S^{(2)}}(s)$ and $r \in C_{S^{(2)}}(s) \cap C_{S^{(2)}}(t)$, one computes that

$$
\begin{gathered}
C_{S^{(2)}}\left(\tau_{33}^{n} I^{2}\right)=C_{S^{(2)}}\left(\tau_{33}^{n} J^{2}\right)=S_{0}^{(2)} \\
C_{S^{(2)}}\left(\tau_{33} I^{2 m} J^{2 m}\right)=S^{(2)}=S_{0}^{(2)} \cup S_{1}^{(2)} \\
C_{S^{(2)}}\left(\tau_{33}^{n} I^{2 m} J^{-2 m} \theta\right)=\left\{\tau_{33}^{p} I^{2 q} J^{-2 q} \theta, \quad \tau_{33} I^{2 p} J^{2 p} ; 0 \leq p, q \leq 1\right\} \\
C_{S^{(2)}}\left(\tau_{33}^{n} I^{2 m+1} J^{-2 m-1} \theta\right)=\left\{\tau_{33}^{p} I^{2 q+1} J^{-2 q-1} \theta, \quad \tau_{33} I^{2 p} J^{2 p} ; 0 \leq p, q \leq 1\right\} .
\end{gathered}
$$

Any subgroup $\mathbb{Z}_{2} \times \mathbb{Z}_{2} \times \mathbb{Z}_{2} \simeq H \subset\{\operatorname{Id}\} \cup S_{0}^{(2)} \cup S_{1}^{(2)}$ intersects $S_{1}^{(2)}$, due to $\left|S_{0}^{(2)}\right|=6$. That allows to assume that $s \in S_{1}^{(2)}$ and observe that

$$
C_{S^{(2)}}(s)=\left\{s, \quad(-\mathrm{Id}) s, \tau_{33} s, \quad \tau_{33}(-\mathrm{Id}) s, \tau_{33}, \quad \tau_{33}(-\mathrm{Id})\right\} .
$$


If $t=\tau_{33} I^{2 p} J^{2 p} \in C_{S^{(2)}}(s)$ then $C_{S^{(2)}}(t)=S^{(2)}$, so that

$$
H \backslash\{\operatorname{Id}, s, t\} \subseteq\left[C_{S^{(2)}}(s) \cap C_{S^{(2)}}(t)\right] \backslash\{s, t\}=C_{S^{(2)}} \backslash\{s, t\}
$$

with $5=|H \backslash\{\operatorname{Id}, s, t\}| \leq\left|C_{S^{(2)}}(s) \backslash\{s, t\}\right|=4$ is an absurd. For $t \in C_{S^{(2)}}(s) \backslash$ $\left\{\tau_{33} I^{2 p} J^{2 p} ; 0 \leq p \leq 1\right\}$ one has $C_{S^{(2)}}(t)=C_{S^{(2)}}(s)$, which again leads to (2). Therefore, there is no subgroup $\mathbb{Z}_{2} \times \mathbb{Z}_{2} \times \mathbb{Z}_{2} \simeq H \not \supset-\mathrm{Id}$ of $G_{-1}$.

Concerning the non-existence of subgroups - Id $\notin H \subset S$ of order 16 , the abelian -Id $\notin H \subset S$ of order 16 may be isomorphic to $\mathbb{Z}_{4} \times \mathbb{Z}_{4}, \mathbb{Z}_{4} \times \mathbb{Z}_{2} \times \mathbb{Z}_{2}$ or $\mathbb{Z}_{2} \times \mathbb{Z}_{2} \times \mathbb{Z}_{2} \times \mathbb{Z}_{2}$. Any $H \simeq \mathbb{Z}_{4} \times \mathbb{Z}_{4}$ is generated by $s, t \in S^{(4)}$ with $s^{2} \neq t^{2}$. Replacing, eventually, $s$ by $s^{3}$ and $t$ by $t^{3}$, one has $s=\tau_{33}^{n} I J^{2 m}, t=\tau_{33}^{p} I^{2 q} J$ with $0 \leq n, m, p, q \leq 1$. Then $s^{2} t^{2}=I^{2} J^{2}=-\mathrm{Id} \in H$ is an absurd. The groups $H \simeq \mathbb{Z}_{4} \times \mathbb{Z}_{2} \times \mathbb{Z}_{2}$ are generated by $s \in S^{(4)}$ and $t, r i n C_{S^{(2)}}(s)$ with $r \in C_{S^{(2)}}(t)$. In the case of $s=\tau_{33}^{n} I J^{2 m}$, the centralizer $C_{S^{(2)}}(s)=S_{0}^{(2)}$. Bearing in mind that $s^{2}=I^{2}$, one observes that $\langle t, r\rangle \cap\left\{I^{2}, J^{2}\right\}=\emptyset$. Therefore $t, r \in\left\{\tau_{33} I^{2 p} J^{2 q} ; 0 \leq p, q \leq 1\right\}$, whereas $\operatorname{tr} \in\left\{\mathrm{Id}, I^{2}, J^{2},-\mathrm{Id}\right\}$. That reveals the non-existence of $\mathbb{Z}_{4} \times \mathbb{Z}_{2} \times \mathbb{Z}_{2} \simeq H \not \supset-$ Id. The groups $H \simeq \mathbb{Z}_{2} \times \mathbb{Z}_{2} \times \mathbb{Z}_{2} \times \mathbb{Z}_{2}$ contain 15 elements of order two, while $\left|S^{(2)}\right|=14$. Therefore there is no abelian group $-\mathrm{Id} \notin H \leq G_{-1}$ of order 16 .

There are three non-abelian groups of order 16 , which do not contain a non-abelian subgroup of order 8 and consist of elements of order 1,2 or 4 . If

$$
\left\langle s, t ; s^{4}=e, t^{4}=e, s t=t s^{3}\right\rangle \simeq H \subset S
$$

then $s, t \in S^{(4)} \subset G_{-1}^{\prime}=\left\langle\tau_{33}, I, J\right\rangle$ commute and imply that $s$ is of order two. The assumption

$$
\left\langle a, b, c ; a^{4}=e, b^{2}=e, c^{2}=e, c b c a^{2} b=e, b a=a b, c a=a c\right\rangle \simeq H \subset S
$$

requires $b, c \in C_{S^{(2)}}(a)=S_{0}^{(2)}=\left\{\tau_{33} I^{2 m} J^{2 l}, I^{2}, J^{2} ; 0 \leq m, l \leq 1\right\}$. Then $b$ and $c$ commute and imply that $c b c a^{2} b=e=a^{2}=e$. Finally, for

$$
G_{4,4}=\left\langle s, t ; s^{4}=e, t^{4}=e, s t s t=e, t s^{3}=s t^{3}\right\rangle
$$

there follows $s, t \in S^{(4)} \subset G_{-1}^{\prime}$, whereas $s t=t s$. Consequently, $s^{2}=t^{2}$ and $G_{4,4}=\left\{s^{i} t^{j} ; 0 \leq i \leq 3,0 \leq j \leq 1\right\}$ is of order $\leq 8$, contrary to $\left|G_{4,4}\right|=16$. Thus, there is no subgroup - Id $\notin H \leq G_{-1}$ of order 16 .

Throughout, we use the notations $H_{\alpha}^{\beta}(\gamma)$ from Lemma 16 and denote by $\Gamma_{\alpha}^{\beta}(\gamma)$ the corresponding lattices with $\Gamma_{\alpha}^{\beta}(\gamma) / \Gamma_{-1}^{(6,8)}=H_{\alpha}^{\beta}(\gamma)$.

Theorem 17. For the groups $H=H_{4 \times 2}^{\prime}(p, q)=\left\langle I J^{2 p}, \tau_{33} J^{2 q}\right\rangle, H_{4 \times 2}^{\prime \prime}(p, q)=$ $\left\langle I^{2 p} J, \tau_{33} I^{2 q}\right\rangle, H_{4}^{\prime}(1-m, m)=\left\langle\tau_{33}^{1-m} I J^{2 m}\right\rangle, H_{4}^{\prime \prime}(1-m, m)=\left\langle\tau_{33}^{1-m} I^{2 m} J\right\rangle$, 
$H_{2 \times 2}^{\prime}(1)=\left\langle\tau_{33} J^{2}, I^{2}\right\rangle, H_{2 \times 2}^{\prime \prime}(1)=\left\langle\tau_{33} I^{2}, J^{2}\right\rangle, H_{2 \times 2}^{\theta}(n, m)=\left\langle\tau_{33}^{n} I^{m} J^{-m} \theta\right.$, $\left.\tau_{33} I^{2} J^{2}\right\rangle$ with $0 \leq p, q \leq 1,(p, q) \neq(1,1)$ and $0 \leq n, m \leq 1$ the logarithmiccanonical map

$$
\Phi^{H}: \widehat{\mathbb{B} / \Gamma_{H}} \rightarrow \mathbb{P}\left(\left[\Gamma_{H}, 1\right]\right)=\mathbb{P}^{1}
$$

is dominant and not globally defined. The Baily-Borel compactifications $\widehat{\mathbb{B} / \Gamma_{H}}$ are birational to ruled surfaces with elliptic bases whenever $H=H_{4 \times 2}^{\prime}(0,0)$, $H_{4 \times 2}^{\prime \prime}(0,0), H_{4}^{\prime}(1,0)$ or $H_{4}^{\prime \prime}(1,0)$. The remaining ones are rational surfaces.

Proof: According to Lemma 11(v), it suffices to prove the theorem for $H_{4 \times 2}^{\prime}(p, q)$ with $(p, q) \neq(1,1), H_{4}^{\prime}(1-m, m) H_{2 \times 2}^{\prime}(1)$ and $H_{2 \times 2}^{\theta}(n, m)$. If $H=H_{4}^{\prime}(1,0)=\left\langle\tau_{33} I\right\rangle$, then $\mathcal{L}^{H}$ is generated by $1 \in \mathbb{C}$ and Reynolds operators

$$
\begin{gathered}
R_{H}\left(f_{56}\right)=0, R_{H}\left(f_{78}\right)=0, \quad R_{H}\left(f_{157}\right)=f_{157}-\mathrm{e}^{\frac{\pi}{2}} f_{258}+\mathrm{ie}^{\frac{\pi}{2}} f_{357}+\mathrm{i} f_{458} \\
R_{H}\left(f_{168}\right)=f_{168}-\mathrm{i} f_{267}+\mathrm{ie}^{-\frac{\pi}{2}} f_{368}+\mathrm{e}^{-\frac{\pi}{2}} f_{467}=\mathrm{ie}^{-\frac{\pi}{2}} R_{H}\left(f_{368}\right) .
\end{gathered}
$$

There are four $\Gamma_{4}^{\prime}(1,0)$-cusps : $\bar{\kappa}_{1}=\bar{\kappa}_{2}=\bar{\kappa}_{3}=\bar{\kappa}_{4}, \bar{\kappa}_{5}, \bar{\kappa}_{6}, \bar{\kappa}_{7}=\bar{\kappa}_{8}$. Applying Lemma 11 ii) to $T_{1} \subset\left(R_{H}\left(f_{157}\right)\right)_{\infty}, R_{H}\left(f_{168}\right)_{\infty} \subseteq \sum_{i=1}^{8} T_{i}$, one concludes that $R_{H}\left(f_{168}\right) \in \operatorname{Span}_{\mathbb{C}}\left(1, R_{H}\left(f_{157}\right)\right.$. Therefore $\mathcal{L}^{H} \simeq \mathbb{C}^{2}$ and $\Phi^{H_{4}^{\prime}(1,0)}$ is a dominant map to $\mathbb{P}\left(\mathcal{L}^{H}\right) \simeq \mathbb{P}^{1}$. Since $\left.R_{H}\left(f_{157}\right)\right|_{T_{6}} \neq \infty$, the entire $\left[\Gamma_{4}^{\prime}(1,0), 1\right]$ vanishes at $\bar{\kappa}_{6}$ and $\Phi^{H_{4}^{\prime}(1,0)}$ is not defined at $\bar{\kappa}_{6}$.

The group $H=H_{4 \times 2}^{\prime}(0,0)=\left\langle I, \tau_{33}\right\rangle$ contains $F=H_{4}^{\prime}(1,0)$ as a subgroup of index two with non-trivial coset representative $I$. Therefore $R_{H}\left(f_{56}\right)=R_{F}\left(f_{56}\right)+$ $I R_{F}\left(f_{56}\right)=0, R_{H}\left(f_{78}\right)=0$ and $\operatorname{rk} \Phi^{H_{4 \times 2}^{\prime}(0,0)} \leq 1$. Due to

$R_{H}\left(f_{157}\right)=f_{157}-\mathrm{ie}^{\frac{\pi}{2}} f_{168}-\mathrm{e}^{\frac{\pi}{2}} f_{258}-\mathrm{e}^{\frac{\pi}{2}} f_{267}+f_{368}+\mathrm{ie} \frac{\pi}{2} f_{357}+\mathrm{i} f_{458}-\mathrm{i} f_{467}$ $\mathcal{L}^{H}=\operatorname{Span}_{\mathbb{C}}\left(1, R_{H}\left(f_{157}\right)\right)$. Lemma 13 provides $\left.\frac{f_{157}-\mathrm{ie}^{\frac{\pi}{2}} f_{168}}{\Sigma_{1}}\right|_{T_{1}}=-2 \mathrm{ie}^{-\frac{\pi}{2}} \neq 0$, whereas $\left.R_{H}\left(f_{157}\right)\right|_{T_{1}}=\infty$. Therefore $\operatorname{dim}_{\mathbb{C}} \mathcal{L}^{H}=2$ and $\Phi^{H_{4 \times 2}^{\prime}(0,0)}$ is a dominant map to $\mathbb{P}^{1}$. The $\Gamma_{4 \times 2}(0,0)$-cusps are $\bar{\kappa}_{1}=\bar{\kappa}_{2}=\bar{\kappa}_{3}=\bar{\kappa}_{4}, \bar{\kappa}_{5}=\bar{\kappa}_{6}$ and $\bar{\kappa}_{7}=\bar{\kappa}_{8}$. Again from Lemma 13, $\left.\frac{f_{157}-\mathrm{e}^{\frac{\pi}{2}} f_{258}+\mathrm{ie}^{\frac{\pi}{2}} f_{357}+\mathrm{i} f_{458}}{\Sigma_{5}}\right|_{T_{5}}=0$, so that $R_{H}\left(f_{157}\right)$ is regular over $T_{5}+T_{6}$. As a result, $\Phi^{H_{4 \times 2}^{\prime}(0,0)}$ is not defined at $\bar{\kappa}_{5}=\bar{\kappa}_{6}$.

For $H=H_{4}^{\prime}(0,1)=\left\langle I J^{2}\right\rangle$, the space $\mathcal{L}^{H}$ is spanned by 1 and Reynolds operators

$$
\begin{gathered}
R_{H}\left(f_{56}\right)=0, \quad R_{H}\left(f_{78}\right)=0, \quad R_{H}\left(f_{157}\right)=f_{157}+\mathrm{e}^{\frac{\pi}{2}} f_{267}+\mathrm{ie}^{\frac{\pi}{2}} f_{357}+\mathrm{i} f_{467} \\
R_{H}\left(f_{168}\right)=f_{168}+\mathrm{i} f_{258}+\mathrm{ie}^{-\frac{\pi}{2}} f_{368}+\mathrm{e}^{-\frac{\pi}{2}} f_{458}=\mathrm{i} R_{H}\left(f_{258}\right) .
\end{gathered}
$$


The $\Gamma_{4}^{\prime}(0,1)$-cusps are $\bar{\kappa}_{1}=\bar{\kappa}_{2}=\bar{\kappa}_{3}=\bar{\kappa}_{4}, \bar{\kappa}_{5}=\bar{\kappa}_{6}, \bar{\kappa}_{7}$ and $\bar{\kappa}_{8}$. Note that $T_{1} \subset\left(R_{H}\left(f_{157}\right)\right)_{\infty},\left(R_{H}\left(f_{168}\right)\right)_{\infty} \subseteq \sum_{i=1}^{8} T_{i}$, in order to conclude that $R_{H}\left(f_{168}\right) \in$ $\operatorname{Span}_{\mathbb{C}}\left(1, R_{H}\left(f_{157}\right)\right)$ by Lemma 11 ii). Therefore $\mathcal{L}^{H}=\operatorname{Span}_{\mathbb{C}}\left(1, R_{H}\left(f_{157}\right)\right) \simeq$ $\mathbb{C}^{2}$ and $\Phi^{H_{4}^{\prime}(0,1)}$ is a dominant map to $\mathbb{P}^{1}$. Lemma 13 supplies $\left.\frac{f_{157}+\mathrm{ie}^{\frac{\pi}{2}} f_{357}}{\Sigma_{5}}\right|_{T_{5}}=0$ and justifies that $\Phi^{H_{4}^{\prime}(0,1)}$ is not defined at $\bar{\kappa}_{5}$.

For $H=H_{4 \times 2}^{\prime}(1,0)=\left\langle I J^{2}, \tau_{33}\right\rangle$ note that $R_{H}\left(f_{56}\right)=0, R_{H}\left(f_{78}\right)=0$, as far as $H_{4}^{\prime}(1,0)$ is a subgroup of $H_{4 \times 2}^{\prime}(1,0)$. Further,

$R_{H}\left(f_{157}\right)=f_{157}-\mathrm{ie}^{\frac{\pi}{2}} f_{168}+\mathrm{e}^{\frac{\pi}{2}} f_{267}+\mathrm{e}^{\frac{\pi}{2}} f_{258}+\mathrm{ie}{ }^{\frac{\pi}{2}} f_{357}+f_{368}+\mathrm{i} f_{467}-\mathrm{i} f_{458}$

has a pole over $\sum_{i=1}^{4} T_{i}$, according to $\left.\frac{f_{157}-\mathrm{ie}^{\frac{\pi}{2}} f_{168}}{\Sigma_{1}}\right|_{T_{1}}=-2 \mathrm{ie}^{-\frac{\pi}{2}} \neq 0$ by Lemma 13 and the transitiveness of the $H_{4}^{\prime}(1,0)$-action on $\left\{\kappa_{i} ; 1 \leq i \leq 4\right\}$. Therefore $\mathcal{L}^{H}=\operatorname{Span}_{\mathbb{C}}\left(1, R_{H}\left(f_{157}\right)\right) \simeq \mathbb{C}^{2}$ and $\Phi^{H_{4 \times 2}^{\prime}(1,0)}$ is a dominant map to $\mathbb{P}^{1}$. One computes immediately that the $\Gamma_{4 \times 2}^{\prime}(1,0)$-cusps are $\bar{\kappa}_{1}=\bar{\kappa}_{2}=\bar{\kappa}_{3}=\bar{\kappa}_{4}, \bar{\kappa}_{5}=\bar{\kappa}_{6}$ and $\bar{\kappa}_{7}=\bar{\kappa}_{8}$. Again from Lemma 13, $\left.\frac{f_{157}+\mathrm{e}^{\frac{\pi}{2}} f_{258}+\mathrm{e}^{\frac{\pi}{2}} f_{357}-\mathrm{i} f_{458}}{\Sigma_{5}}\right|_{T_{5}}=0, R_{H}\left(f_{157}\right)$ has no pole at $T_{5}+T_{6}$ and $\Phi^{H_{4 \times 2}^{\prime}(1,0)}$ is not defined at $\bar{\kappa}_{5}=\bar{\kappa}_{6}$.

If $H=H_{2 \times 2}^{\prime}(1)=\left\langle I^{2}, \tau_{33} J^{2}\right\rangle$ then

$$
\begin{gathered}
R_{H}\left(f_{56}\right)=0, R_{H}\left(f_{78}\right)=4 f_{78}, \quad R_{H}\left(f_{157}\right)=f_{157}+\mathrm{ie}^{\frac{\pi}{2}} f_{168}+\mathrm{ie}^{\frac{\pi}{2}} f_{357}-f_{368} \\
R_{H}\left(f_{258}\right)=f_{258}-f_{267}-\mathrm{ie}^{-\frac{\pi}{2}} f_{467}-\mathrm{ie}^{-\frac{\pi}{2}} f_{458} \quad \text { and } \quad 1 \in \mathbb{C}
\end{gathered}
$$

$\operatorname{span} \mathcal{L}^{H}$. The $\Gamma_{2 \times 2}^{\prime}(1)$-cusps are $\bar{\kappa}_{1}=\bar{\kappa}_{3}, \bar{\kappa}_{2}=\bar{\kappa}_{4}, \bar{\kappa}_{5}=\bar{\kappa}_{6}$ and $\bar{\kappa}_{7}=$ $\bar{\kappa}_{8}$. Lemma 13 reveals that $\left.\frac{f_{157}+\mathrm{ie}^{\frac{\pi}{2}} f_{168}}{\Sigma_{1}}\right|_{T_{1}}=\left.\frac{\mathrm{ie}^{\frac{\pi}{2}} f_{357}-f_{368}}{\Sigma_{3}}\right|_{T_{3}}=\left.\frac{f_{258}-f_{267}}{\Sigma_{2}}\right|_{T_{2}}=$ $\left.\frac{f_{467}+f_{458}}{\Sigma_{4}}\right|_{T_{4}}=0$, so that $R_{H}\left(f_{157}\right), R_{H}\left(f_{258}\right) \in \operatorname{Span}_{\mathbb{C}}\left(1, f_{78}\right)$ and $\mathcal{L}^{H} \simeq \mathbb{C}^{2}$. As a result, $\Phi^{H_{2 \times 2}^{\prime}(1)}$ is a dominant map to $\mathbb{P}^{1}$, which is not defined at $\bar{\kappa}_{1}$ and $\bar{\kappa}_{2}$. For the group $H=H_{4 \times 2}^{\prime}(0,1)=\left\langle I, \tau_{33} J^{2}\right\rangle$, containing $H_{2 \times 2}^{\prime}(1)=\left\langle I^{2}, \tau_{33} J^{2}\right\rangle$ there follows $R_{H}\left(f_{56}\right)=0$ and $\operatorname{rk} \Phi^{H_{4 \times 2}^{\prime}(0,1)} \leq 1$. Therefore $R_{H}\left(f_{78}\right)=8 f_{78}$, $R_{H}\left(f_{157}\right)=f_{157}+\mathrm{ie}^{\frac{\pi}{2}} f_{168}+\mathrm{e}^{\frac{\pi}{2}} f_{258}-\mathrm{e}^{\frac{\pi}{2}} f_{267}+\mathrm{ie}^{\frac{\pi}{2}} f_{357}-f_{368}-\mathrm{i} f_{458}-\mathrm{i} f_{467}$ and $1 \in \mathbb{C} \operatorname{span} \mathcal{L}^{H}$. The $\Gamma_{4 \times 2}^{\prime}(0,1)$-cusps are $\bar{\kappa}_{1}=\bar{\kappa}_{2}=\bar{\kappa}_{3}=\bar{\kappa}_{4}, \bar{\kappa}_{5}=$ $\bar{\kappa}_{6}$ and $\bar{\kappa}_{7}=\bar{\kappa}_{8}$. By Lemma 13, $\left.\frac{f_{157}+\mathrm{ie}^{\frac{\pi}{2}} f_{168}}{\Sigma_{1}}\right|_{T_{1}}=0$, so that $R_{H}\left(f_{157}\right) \in$ $\operatorname{Span}_{\mathbb{C}}\left(1, f_{78}\right) \simeq \mathbb{C}^{2}$. Thus, $\Phi^{H_{4 \times 2}^{\prime}(0,1)}$ is a dominant map to $\mathbb{P}^{1}$, which is not defined at $\bar{\kappa}_{1}$. 
If $H=H_{2 \times 2}^{\theta}(0,0)=\left\langle\theta, \tau_{33} I^{2} J^{2}\right\rangle$ then $\mathcal{L}^{H}$ is spanned by $1 \in \mathbb{C}$,

$$
R_{H}\left(f_{56}\right)=2\left(f_{56}+f_{78}\right), R_{H}\left(f_{157}\right)=f_{157}+\mathrm{ie}^{\frac{\pi}{2}} f_{168}-\mathrm{e}^{\frac{\pi}{2}} f_{357}-\mathrm{i} f_{368}
$$

and $R_{H}\left(f_{467}\right)=2\left(f_{467}+f_{458}\right)$, due to $R_{H}\left(f_{258}\right)=0$. The $\Gamma_{2}^{\theta}(0,0)$-cusps are $\bar{\kappa}_{1}=\bar{\kappa}_{3}, \bar{\kappa}_{2}, \bar{\kappa}_{4}$ and $\bar{\kappa}_{5}=\bar{\kappa}_{6}=\bar{\kappa}_{7}=\bar{\kappa}_{8}$. Lemma 13 provides $\left.\frac{f_{157}+\mathrm{ie}^{\frac{\pi}{2}} f_{168}}{\Sigma_{1}}\right|_{T_{1}}=0$, $\left.\frac{f_{467}+f_{458}}{\Sigma_{4}}\right|_{T_{4}}=0$, whereas $R_{H}\left(f_{157}\right), R_{H}\left(f_{467}\right) \in \operatorname{Span}_{\mathbb{C}}\left(1, R_{H}\left(f_{56}\right)\right) \simeq \mathbb{C}^{2}$. Therefore $\Phi^{H_{2}^{\theta}(0,0)}$ is a dominant map to $\mathbb{P}^{1}$, which is not defined at $\bar{\kappa}_{1}, \bar{\kappa}_{2}$ and $\bar{\kappa}_{4}$. For $H=H_{2 \times 2}^{\theta}(0,1)=\left\langle I J^{-1} \theta, \tau_{33} I^{2} J^{2}\right\rangle$ one has

$$
R_{H}\left(f_{56}\right)=2\left(f_{56}+\mathrm{i} f_{78}\right), \quad R_{H}\left(f_{157}\right)=0, \quad R_{H}\left(f_{168}\right)=0
$$

$R_{H}\left(f_{368}\right)=2\left(f_{368}-\mathrm{ie}^{\frac{\pi}{2}} f_{357}\right), R_{H}\left(f_{258}\right)=f_{258}-f_{267}-\mathrm{e}^{-\frac{\pi}{2}} f_{458}-\mathrm{e}^{-\frac{\pi}{2}} f_{467}$. The $\Gamma_{2 \times 2}^{\theta}(0,1)$-cusps are $\bar{\kappa}_{1}, \overline{\kappa_{3}}, \overline{\kappa_{2}}=\overline{\kappa_{4}}, \overline{\kappa_{5}}=\overline{\kappa_{6}}=\overline{\kappa_{7}}=\overline{\kappa_{8}}$. Lemma 13 implies that $\left.\frac{f_{368}-\mathrm{ie}^{\frac{\pi}{2}} f_{357}}{\Sigma_{3}}\right|_{T_{3}}=0,\left.\frac{f_{258}-f_{267}}{\Sigma_{2}}\right|_{T_{2}}=0,\left.\frac{f_{458}+f_{467}}{\Sigma_{4}}\right|_{T_{4}}=0$, whereas $R_{H}\left(f_{368}\right), R_{H}\left(f_{258}\right) \in \operatorname{Span}_{\mathbb{C}}\left(1, R_{H}\left(f_{56}\right)\right) \simeq \mathbb{C}$. Consequently, $\Phi^{H_{2 \times 2}^{\theta}(0,1)}$ is a dominant map to $\mathbb{P}^{1}$, which is not defined at $\bar{\kappa}_{1}, \bar{\kappa}_{2}$ and $\bar{\kappa}_{4}$.

In the case of $H=H_{2 \times 2}^{\theta}(1,0)=\left\langle\tau_{33} \theta, \tau_{33} I^{2} J^{2}\right\rangle$, the Reynolds operators are

$$
\begin{gathered}
R_{H}\left(f_{56}\right)=2\left(f_{56}-f_{78}\right), \quad R_{H}\left(f_{157}\right)=f_{157}+\mathrm{ie}^{\frac{\pi}{2}} f_{168}+\mathrm{i} f_{368}+\mathrm{e}^{\frac{\pi}{2}} f_{357} \\
R_{H}\left(f_{258}\right)=2\left(f_{258}-f_{267}\right), \quad R_{H}\left(f_{458}\right)=0, \quad R_{H}\left(f_{467}\right)=0 .
\end{gathered}
$$

The $\Gamma_{2 \times 2}^{\theta}(1,0)$-cusps are $\bar{\kappa}_{1}, \bar{\kappa}_{3}, \bar{\kappa}_{2}=\bar{\kappa}_{4}$ and $\bar{\kappa}_{5}=\bar{\kappa}_{6}=\bar{\kappa}_{7}=\bar{\kappa}_{8}$. Lemma 13 yields $\left.\frac{f_{157}+\mathrm{ie}^{\frac{\pi}{2}} f_{168}}{\Sigma_{1}}\right|_{T_{1}}=\left.\frac{\mathrm{i} f_{368}+\mathrm{e}^{\frac{\pi}{2}} f_{357}}{\Sigma_{3}}\right|_{T_{3}}=\left.\frac{f_{258}-f_{267}}{\Sigma_{2}}\right|_{T_{2}}=0$. Consequently, $R_{H}\left(f_{157}\right), R_{H}\left(f_{258}\right) \in \operatorname{Span}_{\mathbb{C}}\left(1, R_{H}\left(f_{56}\right)\right)$. Bearing in mind that $\left.R_{H}\left(f_{56}\right)\right|_{T_{5}}=$ $\infty$, one concludes that $\Phi^{H_{2 \times 2}^{\theta}(1,0)}$ is a dominant map to $\mathbb{P}^{1}$, which is not defined at $\bar{\kappa}_{1}, \bar{\kappa}_{2}$ and $\bar{\kappa}_{3}$.

Finally, for $H=H_{2 \times 2}^{\theta}(1,1)=\left\langle\tau_{33} I J^{-1} \theta, \tau_{33} I^{2} J^{2}\right\rangle$ one has

$$
\begin{gathered}
R_{H}\left(f_{56}\right)=2\left(f_{56}-\mathrm{i} f_{78}\right), R_{H}\left(f_{157}\right)=2\left(f_{157}+\mathrm{ie}^{\frac{\pi}{2}} f_{168}\right), R_{H}\left(f_{357}\right)=0 \\
R_{H}\left(f_{368}\right)=0 \text { and } R_{H}\left(f_{258}\right)=f_{258}-f_{267}+\mathrm{e}^{-\frac{\pi}{2}} f_{467}+\mathrm{e}^{-\frac{\pi}{2}} f_{458} .
\end{gathered}
$$

The $\Gamma_{2 \times 2}^{\theta}(1,1)$-cusps are $\bar{\kappa}_{1}, \bar{\kappa}_{3}, \bar{\kappa}_{2}=\bar{\kappa}_{4}$ and $\bar{\kappa}_{5}=\bar{\kappa}_{6}=\bar{\kappa}_{7}=\bar{\kappa}_{8}$. Lemma 13 implies that $\left.\frac{f_{157}+\mathrm{ie}^{\frac{\pi}{2}} f_{168}}{\Sigma_{1}}\right|_{T_{1}}=\left.\frac{f_{258}-f_{267}}{\Sigma_{2}}\right|_{T_{2}}=0$, so that $R_{H}\left(f_{157}\right), R_{H}\left(f_{258}\right) \in$ $\operatorname{Span}_{\mathbb{C}}\left(1, R_{H}\left(f_{56}\right)\right) \simeq \mathbb{C}^{2}$. As a result, $\Phi^{H_{2 \times 2}^{\theta}(1,1)}$ is a dominant map to $\mathbb{P}^{1}$, which is not defined at $\bar{\kappa}_{1}, \bar{\kappa}_{3}$ and $\bar{\kappa}_{2}$. 
Theorem 18. If $H=H_{2 \times 2}^{\prime}(0)=\left\langle\tau_{33}, I^{2}\right\rangle, H_{2 \times 2}^{\prime \prime}(0)=\left\langle\tau_{33}, J^{2}\right\rangle, H_{2 \times 2}^{\theta}(n)=$ $\left\langle I^{n} J^{-n} \theta, \tau_{33}\right\rangle$ with $0 \leq n \leq 1, H_{4}^{\prime}(n, n)=\left\langle\tau_{33}^{n} I J^{2 n}\right\rangle, H_{4}^{\prime \prime}(n, n)=\left\langle\tau_{33}^{n} I^{2 n} J\right\rangle$ with $0 \leq n \leq 1$ or $H_{2}(1,1)=\left\langle\tau_{33} I^{2} J^{2}\right\rangle$ then the logarithmic-canonical map

$$
\Phi^{H}: \widehat{\mathbb{B} / \Gamma_{H}} \cdots \mathbb{P}\left(\left[\Gamma_{H}, 1\right]\right)=\mathbb{P}^{2}
$$

is dominant and not globally defined. The surface $\widehat{\mathbb{B} / \Gamma_{H}}$ is $\mathrm{K} 3$ for $H=H_{2}(1,1)$, rational for $H=H_{4}^{\prime}(1,1), H_{4}^{\prime \prime}(1,1)$ and ruled with an elliptic base for all the other aforementioned $H$.

Proof: By Lemma $11 \mathrm{v})$, it suffices to consider $H_{2 \times 2}^{\prime}(0), H_{2 \times 2}^{\theta}(n), H_{4}^{\prime}(n, n)$ and $H_{2}(1,1)$.

In the case of $H=H_{2 \times 2}^{\prime}(0)=\left\langle\tau_{33}, I^{2}\right\rangle, \mathcal{L}^{H}$ is spanned by

$$
\begin{gathered}
R_{H}\left(f_{56}\right)=0, \quad R_{H}\left(f_{78}\right)=0, \quad R_{H}\left(f_{157}\right)=f_{157}-\mathrm{ie}^{\frac{\pi}{2}} f_{168}+\mathrm{ie}^{\frac{\pi}{2}} f_{357}+f_{368} \\
R_{H}\left(f_{258}\right)=f_{258}+f_{267}-\mathrm{ie}^{-\frac{\pi}{2}} f_{458}+\mathrm{ie}^{-\frac{\pi}{2}} f_{467} \quad \text { and } \quad 1 \in \mathbb{C} .
\end{gathered}
$$

The $\Gamma_{2 \times 2}^{\prime}(0)$-cusps are $\bar{\kappa}_{1}=\bar{\kappa}_{3}, \bar{\kappa}_{2}=\bar{\kappa}_{4}, \bar{\kappa}_{5}=\bar{\kappa}_{6}$ and $\bar{\kappa}_{7}=\bar{\kappa}_{8}$. Lemma 13 provides $\left.\frac{f_{157}-\mathrm{ie} \mathrm{e}^{\frac{\pi}{2}} f_{168}}{\Sigma_{1}}\right|_{T_{1}}=-2 \mathrm{ie}^{-\frac{\pi}{2}} \neq 0$, whereas $\left.R_{H}\left(f_{157}\right)\right|_{T_{1}}=\infty$. Similarly, $\left.\frac{f_{258}+f_{267}}{\Sigma_{2}}\right|_{T_{2}}=2 \mathrm{e}^{-\pi} \neq 0$ suffices for $\left.R_{H}\left(f_{258}\right)\right|_{T_{2}}=\infty$. Therefore 1, $R_{H}\left(f_{157}\right), R_{H}\left(f_{258}\right)$ are linearly independent, according to Lemma 11 iii) and constitute a $\mathbb{C}$-basis for $\mathcal{L}^{H}$. In order to assert that $\operatorname{rk} \Phi^{H_{2 \times 2}^{\prime}(0)}=2$, we use that $\left.R_{H}\left(f_{258}\right)\right|_{T_{2}}=\infty$ and $\left.R_{H}\left(f_{157}\right)\right|_{T_{2}} \not \equiv$ const by Lemma 14 with $c=\mathrm{ie}^{\frac{\pi}{2}}$. Lemma 13 provides $\left.\frac{f_{157}+\mathrm{e}^{\frac{\pi}{2}} f_{357}}{\Sigma_{5}}\right|_{T_{5}}=0$, in order to conclude that $\left.R_{H}\left(f_{157}\right)\right|_{T_{5}} \neq$ $\infty$ and the entire $\left[\Gamma_{2 \times 2}^{\prime}(0), 1\right]$ vanishes at $\bar{\kappa}_{5}$. Therefore $\Phi^{H_{2 \times 2}^{\prime}(0)}$ is a dominant map to $\mathbb{P}\left(\left[\Gamma_{2 \times 2}^{\prime}(0), 1\right]\right)=\mathbb{P}^{2}$, which is not defined at $\bar{\kappa}_{5}$.

For $H=H_{2 \times 2}^{\theta}(0)=\left\langle\theta, \tau_{33}\right\rangle$, the Reynolds operators are

$$
\begin{aligned}
R_{H}\left(f_{56}\right)=0, \quad R_{H}\left(f_{78}\right) & =0, \quad R_{H}\left(f_{157}\right)=f_{157}-\mathrm{ie}^{\frac{\pi}{2}} f_{168}-\mathrm{e}^{\frac{\pi}{2}} f_{357}+\mathrm{i} f_{368} \\
R_{H}\left(f_{258}\right) & =2\left(f_{258}+f_{267}\right), \quad R_{H}\left(f_{467}\right)=0
\end{aligned}
$$

generate $\mathcal{L}^{H}$. The $\Gamma_{2 \times 2}^{\theta}(0)$-cusps are $\bar{\kappa}_{1}=\bar{\kappa}_{3}, \bar{\kappa}_{2}, \bar{\kappa}_{4}$ and $\bar{\kappa}_{5}=\bar{\kappa}_{6}=\bar{\kappa}_{7}=\bar{\kappa}_{8}$. According to Lemma 13, $\left.\frac{f_{157}-\mathrm{ie}^{\frac{\pi}{2}} f_{168}}{\Sigma_{1}}\right|_{T_{1}}=-2 \mathrm{ie}^{-\frac{\pi}{2}} \neq 0$, so that $\left.R_{H}\left(f_{157}\right)\right|_{T_{1}}=\infty$. Further, $\left.\frac{f_{258}+f_{267}}{\Sigma_{2}}\right|_{T_{2}}=2 \mathrm{e}^{-\pi} \neq 0$ and the lemma provides $\left.R_{H}\left(f_{258}\right)\right|_{T_{2}}=\infty$. Therefore $1, R_{H}\left(f_{157}\right), R_{H}\left(f_{258}\right)$ are linearly independent and $\mathcal{L}^{H} \simeq \mathbb{C}^{3}$ by Lemma 11 iii). We claim that

$$
\left.R_{H}\left(f_{258}\right)\right|_{T_{1}}=-2 \mathrm{e}^{-\pi \mathrm{i} v} \frac{\sigma\left((1+\mathrm{i}) v+\omega_{3}\right)}{\sigma((1+\mathrm{i}) v)}\left[\frac{\sigma\left(v-\omega_{1}\right)^{2}}{\sigma\left(v-\omega_{2}\right)^{2}}+\mathrm{e}^{2 \pi(1+\mathrm{i}) v} \frac{\sigma\left(v-\omega_{2}\right)^{2}}{\sigma\left(v-\omega_{1}\right)^{2}}\right]
$$


is non-constant. On one hand, $\left.R_{H}\left(f_{258}\right)\right|_{T_{1}}$ has no poles on $\mathbb{C} \backslash \mathbb{Q}(\mathrm{i})$. On the other hand, $\left.\left[\left.\frac{1}{2} R_{H}\left(f_{258}\right)\right|_{T_{1}}\right] \sigma((1+\mathrm{i}) v)\right|_{v=0}=-\sigma\left(\omega_{3}\right)\left[\frac{1}{\mathrm{i}^{2}}+\mathrm{i}^{2}\right] \neq 0$, so that $\lim _{v \rightarrow 0}\left[\left.R_{H}\left(f_{258}\right)\right|_{T_{1}}\right]=\infty$. According to Lemma $\left.11 \mathrm{iv}\right),\left.R_{H}\left(f_{157}\right)\right|_{T_{1}}=\infty$ and $\left.R_{H}\left(f_{258}\right)\right|_{T_{1}} \not \equiv$ const suffice for $\Phi^{H_{2 \times 2}^{\theta}(0)}$ to be a dominant map to $\mathbb{P}^{2}$. The entire $\mathcal{L}^{H}$ takes finite values on $T_{4}$, so that $\Phi^{H_{2 \times 2}^{\theta}(0)}$ is not defined at $\bar{\kappa}_{4}$.

Concerning $H=H_{2 \times 2}^{\theta}(1)=\left\langle I J^{-1} \theta, \tau_{33}\right\rangle$, one computes that

$$
\begin{gathered}
R_{H}\left(f_{56}\right)=0, \quad R_{H}\left(f_{78}\right)=0, \quad R_{H}\left(f_{157}\right)=2\left(f_{157}-\mathrm{ie}^{\frac{\pi}{2}} f_{168}\right) \\
R_{H}\left(f_{368}\right)=0, \quad R_{H}\left(f_{258}\right)=f_{258}+f_{267}-\mathrm{e}^{-\frac{\pi}{2}} f_{458}+\mathrm{e}^{-\frac{\pi}{2}} f_{467} .
\end{gathered}
$$

The $\Gamma_{2 \times 2}^{\theta}(1)$-cusps are $\bar{\kappa}_{1}, \bar{\kappa}_{3}, \bar{\kappa}_{2}=\bar{\kappa}_{4}$ and $\bar{\kappa}_{5}=\bar{\kappa}_{6}=\bar{\kappa}_{7}=\bar{\kappa}_{8}$. By Lemma 13 we have $\left.\frac{f_{157}-\mathrm{ie}^{\frac{\pi}{2}} f_{168}}{\Sigma_{1}}\right|_{T_{1}}=-2 \mathrm{ie}^{-\frac{\pi}{2}} \neq 0$ and $\left.\frac{f_{258}+f_{267}}{\Sigma_{2}}\right|_{T_{2}}=2 \mathrm{e}^{-\pi} \neq 0$. Therefore $\left.R_{H}\left(f_{157}\right)\right|_{T_{1}}=\infty,\left.R_{H}\left(f_{258}\right)\right|_{T_{2}}=\infty$ and $1, R_{H}\left(f_{157}\right), R_{H}\left(f_{258}\right)$ constitute a $\mathbb{C}$-basis of $\mathcal{L}^{H}$, according to Lemma 11 iii). Applying Lemma 14 with $c=0$, one concludes that $\left.R_{H}\left(f_{157}\right)\right|_{T_{2}} \not \equiv$ const. Then Lemma $\left.11 \mathrm{iv}\right)$ implies that $\Phi^{H_{2 \times 2}^{\theta}(1)}$ is a dominant map to $\mathbb{P}^{2}$. The lack of $f \in \mathcal{L}^{H}$ with $\left.f\right|_{T_{3}}=\infty$ reveals that $\Phi^{H_{2 \times 2}^{\theta}(1)}$ is not defined at $\bar{\kappa}_{3}$.

If $H=H_{4}^{\prime}(0,0)=\langle I\rangle$ then the Reynolds operators are

$$
\begin{gathered}
R_{H}\left(f_{56}\right)=0, R_{H}\left(f_{78}\right)=4 f_{78}, R_{H}\left(f_{157}\right)=f_{157}-\mathrm{e}^{\frac{\pi}{2}} f_{267}+\mathrm{ie}^{\frac{\pi}{2}} f_{357}-\mathrm{i} f_{467} \\
R_{H}\left(f_{168}\right)=f_{168}-\mathrm{i} f_{258}+\mathrm{ie}-\frac{\pi}{2} f_{368}-\mathrm{e}^{-\frac{\pi}{2}} f_{458} \quad \text { and } \quad R_{H}(1)=1 \in \mathbb{C}
\end{gathered}
$$
span $\mathcal{L}^{H}$. The $\Gamma_{4}^{\prime}(0,0)$-cusps are $\bar{\kappa}_{1}=\bar{\kappa}_{2}=\bar{\kappa}_{3}=\bar{\kappa}_{4}, \bar{\kappa}_{5}=\bar{\kappa}_{6}, \bar{\kappa}_{7}$ and $\bar{\kappa}_{8}$. According to Lemma 11 ii), the inclusions $T_{1} \subset\left(R_{H}\left(f_{157}\right)\right)_{\infty},\left(R_{H}\left(f_{168}\right)\right)_{\infty} \subseteq$ $\sum_{i=1}^{8} T_{i}$ suffice for $R_{H}\left(f_{168}\right) \in \operatorname{Span}_{\mathbb{C}}\left(1, R_{H}\left(f_{78}\right), R_{H}\left(f_{157}\right)\right.$. Therefore $\mathcal{L}^{H} \simeq \mathbb{C}^{3}$. Observe that $\left.R_{H}\left(f_{78}\right)\right|_{T_{1}}=4 \Sigma_{12}(v) \not \equiv$ const, in order to apply Lemma $\left.11 \mathrm{iv}\right)$ and assert that $\Phi^{H_{4}^{\prime}(0,0)}$ is a dominant map to $\mathbb{P}^{2}$. As far as $\left.\frac{f_{157}+\mathrm{ie}^{\frac{\pi}{2}} f_{357}}{\Sigma_{5}}\right|_{T_{5}}=0$ by Lemma 13 , the abelian function $R_{H}\left(f_{157}\right)$ has no pole on $T_{5}$. Therefore $\Phi^{H_{4}^{\prime}(0,0)}$ is not defined at $\bar{\kappa}_{5}$.

For $H_{4}^{\prime}(1,1)=\left\langle\tau_{33} I J^{2}\right\rangle$ the Reynolds operators are

$$
\begin{gathered}
R_{h}\left(f_{56}\right)=0, \quad R_{H}\left(f_{78}\right)=4 f_{78}, \quad R_{H}\left(f_{157}\right)=f_{157}+\mathrm{e}^{\frac{\pi}{2}} f_{258}+\mathrm{ie}^{\frac{\pi}{2}} f_{357}-\mathrm{i} f_{458} \\
R_{H}\left(f_{168}\right)=f_{168}+\mathrm{i} f_{267}+\mathrm{ie}-\frac{\pi}{2} f_{368}-\mathrm{e}^{-\frac{\pi}{2}} f_{467} .
\end{gathered}
$$

The $\Gamma_{4}^{\prime}(1,1)$-cusps are $\bar{\kappa}_{1}=\bar{\kappa}_{2}=\bar{\kappa}_{3}=\bar{\kappa}_{4}, \bar{\kappa}_{5}, \bar{\kappa}_{6}$ and $\bar{\kappa}_{7}=\bar{\kappa}_{8}$. Due to $T_{1} \subset\left(R_{H}\left(f_{157}\right)\right)_{\infty},\left(R_{H}\left(f_{168}\right)\right)_{\infty} \subseteq \sum_{i=1}^{8} T_{i}$, Lemma 11 ii) applies to provide 
$R_{H}\left(f_{168}\right) \in \operatorname{Span}_{\mathbb{C}}\left(1, R_{H}\left(f_{78}\right), R_{H}\left(f_{157}\right)\right)$. Thus, $\mathcal{L}^{H} \simeq \mathbb{C}^{3}$. According to Lemma $11 \mathrm{iv}),\left.R_{H}\left(f_{78}\right)\right|_{T_{1}}=4 \Sigma_{12}(v) \not \equiv$ const suffices for $\Phi^{H_{4}^{\prime}(1,1)}$ to be a dominant rational map to $\mathbb{P}^{2}$. Further, $\left.\frac{f_{157}+\mathrm{ie}^{\frac{\pi}{2}} f_{357}}{\Sigma_{5}}\right|_{T_{5}}=0$ by Lemma 13 implies that $R_{H}\left(f_{157}\right)$ has no pole over $T_{5}$ and $\Phi^{H_{4}^{\prime}(1,1)}$ is not defined at $\bar{\kappa}_{5}$.

If $H=H_{2}(1,1)=\left\langle\tau_{33} I^{2} J^{2}\right\rangle$ then $\mathcal{L}^{H}$ is generated by

$$
1 \in \mathbb{C}, \quad R_{H}\left(f_{56}\right)=2 f_{56}, \quad R_{H}\left(f_{78}\right)=2 f_{78}, \quad R_{H}\left(f_{157}\right)=f_{157}+\mathrm{ie}^{\frac{\pi}{2}} f_{168}
$$

$R_{H}\left(f_{368}\right)=f_{368}-\mathrm{ie}^{\frac{\pi}{2}} f_{357}, \quad R_{H}\left(f_{258}\right)=f_{258}-f_{267}, \quad R_{H}\left(f_{467}\right)=f_{467}+f_{458}$.

The $\Gamma_{2}(1,1)$-cusps are $\bar{\kappa}_{1}, \bar{\kappa}_{2}, \bar{\kappa}_{3}, \bar{\kappa}_{4}, \bar{\kappa}_{5}=\bar{\kappa}_{6}$ and $\bar{\kappa}_{7}=\bar{\kappa}_{8}$. By Lemma 13 one has $\left.\frac{f_{157}+\mathrm{ie}^{\frac{\pi}{2}} f_{168}}{\Sigma_{1}}\right|_{T_{1}}=\left.\frac{f_{368}-\mathrm{ie} \mathrm{e}^{\frac{\pi}{2}} f_{357}}{\Sigma_{3}}\right|_{T_{3}}=\left.\frac{f_{258}-f_{267}}{\Sigma_{2}}\right|_{T_{2}}=\left.\frac{f_{467}+f_{458}}{\Sigma_{4}}\right|_{T_{4}}=0$. Thus, $R_{H}\left(f_{157}\right), R_{H}\left(f_{368}\right), R_{H}\left(f_{258}\right), R_{H}\left(f_{467}\right) \in \operatorname{Span}_{\mathbb{C}}\left(1, R_{H}\left(f_{56}\right), R_{H}\left(f_{78}\right)\right)$ and $\mathcal{L}^{H} \simeq \mathbb{C}^{3}$. Bearing in mind that $\left.R_{H}\left(f_{56}\right)\right|_{T_{5}}=\infty,\left.R_{H}\left(f_{78}\right)\right|_{T_{5}} \not \equiv$ const, one applies Lemma $11 \mathrm{iv)}$ and concludes that $\Phi^{H_{2}(1,1)}$ is a dominant map to $\mathbb{P}^{2}$. Since $\mathcal{L}^{H}$ has no pole over $\sum_{i=1}^{4} T_{i}$, the map $\Phi^{H_{2}(1,1)}$ is not defined at $\bar{\kappa}_{1}, \bar{\kappa}_{2}, \bar{\kappa}_{3}, \bar{\kappa}_{4}$.

Let us recall from Hacon and Pardini's [1] that the geometric genus $p_{g}(X)=$ $\operatorname{dim}_{\mathbb{C}} H^{0}\left(X, \Omega_{X}^{2}\right)$ of a smooth minimal surface $X$ of general type is at most 4 . The next theorem provides a smooth toroidal compactification $Y=\left(\mathbb{B} / \Gamma_{\left\langle\tau_{33}\right\rangle}\right)^{\prime}$ with abelian minimal model $A_{-1} /\left\langle\tau_{33}\right\rangle$ and $\operatorname{dim}_{\mathbb{C}} H^{0}\left(Y, \Omega_{Y}^{2}\left(T^{\prime}\right)\right)=5$.

Theorem 19. i) For $H=H_{2}^{\prime}=\left\langle I^{2}\right\rangle, H_{2}^{\prime \prime}=\left\langle J^{2}\right\rangle, H_{2}(n, 1-n)=\left\langle\tau_{33} I^{2 n} J^{2-2 n}\right\rangle$ or $H_{2}^{\theta}(n, k)=\left\langle\tau_{33}^{n} I^{k} J^{-k} \theta\right\rangle$ with $0 \leq n \leq 1,0 \leq k \leq 3$ the logarithmiccanonical map

$$
\Phi^{H}: \widehat{\mathbb{B} / \Gamma_{H}} \rightarrow \rightarrow \mathbb{P}\left(\left[\Gamma_{H}, 1\right]\right)=\mathbb{P}^{3}
$$

has maximal $\operatorname{rk} \Phi^{H}=2$. For $H \neq H_{2}(n, 1-n)$ the rational map $\Phi^{H}$ is not globally defined and $\widehat{\mathbb{B} / \Gamma_{H}}$ are ruled surfaces with elliptic bases. In the case of $H=H_{2}(n, 1-n)$ the surface $\widehat{\mathbb{B} / \Gamma_{H}}$ is hyperelliptic.

ii) For $H=H_{2}(0,0)=\left\langle\tau_{33}\right\rangle$ the smooth surface $\left(\mathbb{B} / \Gamma_{\left\langle\tau_{33}\right\rangle}\right)^{\prime}$ has abelian minimal model $A_{-1} /\left\langle\tau_{33}\right\rangle$ and the logarithmic-canonical map

$$
\Phi^{\left\langle\tau_{33}\right\rangle}: \widehat{\mathbb{B} / \Gamma_{\left\langle\tau_{33}\right\rangle}} \rightarrow \rightarrow \mathbb{P}\left(\left[\Gamma_{\left\langle\tau_{33}\right\rangle}, 1\right]\right)=\mathbb{P}^{4}
$$

is of maximal $\mathrm{rk} \Phi^{\left\langle\tau_{33}\right\rangle}=2$.

Proof: i) By Lemma 11 v), it suffices to prove the statement for $H_{2}^{\prime}, H_{2}(1,0)$ and $H_{2}^{\theta}(n, k)=\left\langle\tau_{33}^{n} I^{k} J^{-k} \theta\right\rangle$ with $0 \leq n \leq 1,0 \leq k \leq 2$. 
Note that $H_{2}^{\prime}, H_{2}(1,0)$ are subgroups of $H_{2 \times 2}^{\prime}(0)=\left\langle\tau_{33}, I^{2}\right\rangle$ and $\operatorname{rk} \Phi^{H_{2 \times 2}^{\prime}(0)}=2$. By Lemma $11 \mathrm{iv}$ ) that suffices for $\operatorname{rk} \Phi^{H_{2}^{\prime}}=\operatorname{rk} \Phi^{H_{2}(1,0)}=2$.

In the case of $H=H_{2}^{\prime}=\left\langle I^{2}\right\rangle$, the Reynolds operators

$$
\begin{array}{cc}
R_{H}\left(f_{56}\right)=0, & R_{H}\left(f_{78}\right)=2 f_{78} \\
R_{H}\left(f_{157}\right)=f_{157}+\mathrm{ie} \frac{\pi}{2} f_{357}, & R_{H}\left(f_{168}\right)=f_{168}+\mathrm{ie}^{-\frac{\pi}{2}} f_{368} \\
R_{H}\left(f_{258}\right)=f_{258}-\mathrm{ie}^{-\frac{\pi}{2}} f_{458}, & R_{H}\left(f_{267}\right)=f_{267}+\mathrm{ie}^{-\frac{\pi}{2}} f_{467} .
\end{array}
$$

The $\Gamma_{2}^{\prime}$-cusps are $\bar{\kappa}_{1}=\bar{\kappa}_{3}, \bar{\kappa}_{2}=\bar{\kappa}_{4}, \bar{\kappa}_{5}, \bar{\kappa}_{6}, \bar{\kappa}_{7}$ and $\bar{\kappa}_{8}$. According to Lemma 11 ii), the inclusions $T_{1} \subset\left(R_{H}\left(f_{157}\right)\right)_{\infty},\left(R_{H}\left(f_{168}\right)\right)_{\infty} \subseteq T_{1}+T_{3}+\sum_{\alpha=5}^{8} T_{\alpha}$ suffice for $R_{H}\left(f_{168}\right) \in \operatorname{Span}_{\mathbb{C}}\left(1, R_{H}\left(f_{78}\right), R_{H}\left(f_{157}\right)\right)$. Similarly, from $T_{2} \subset$ $\left(R_{H}\left(f_{258}\right)\right)_{\infty},\left(R_{H}\left(f_{267}\right)\right)_{\infty} \subseteq T_{2}+T_{4}+\sum_{\alpha=5}^{8} T_{\alpha}$ there follows $R_{H}\left(f_{267}\right) \in$ $\operatorname{Span}_{\mathbb{C}}\left(1, R_{H}\left(f_{78}\right), R_{H}\left(f_{258}\right)\right)$. As a result, one concludes that the space of the invariants $\mathcal{L}^{H}=\operatorname{Span}_{\mathbb{C}}\left(1, R_{H}\left(f_{78}\right), R_{H}\left(f_{157}\right), R_{H}\left(f_{258}\right)\right) \simeq \mathbb{C}^{4}$. Since $\mathcal{L}^{H}$ has no pole over $T_{6}$, the rational map $\Phi^{H_{2}^{\prime}}$ is not defined at $\bar{\kappa}_{6}$.

If $H=H_{2}(1,0)=\left\langle\tau_{33} I^{2}\right\rangle$, then $\mathcal{L}^{H}$ is spanned by

$$
\begin{gathered}
1 \in \mathbb{C}, \quad R_{H}\left(f_{56}\right)=2 f_{56}, \quad R_{H}\left(f_{78}\right)=0 \\
R_{H}\left(f_{157}\right)=f_{157}+f_{368}, \quad R_{H}\left(f_{258}\right)=f_{258}+\mathrm{ie}^{-\frac{\pi}{2}} f_{467} .
\end{gathered}
$$

The $\Gamma_{2}(1,0)$-cusps are $\bar{\kappa}_{1}=\bar{\kappa}_{3}, \bar{\kappa}_{2}=\bar{\kappa}_{4}, \bar{\kappa}_{5}=\bar{\kappa}_{6}, \bar{\kappa}_{7}=\bar{\kappa}_{8}$. According to Lemma 11 iii), the inclusions $T_{1}+T_{3} \subset\left(R_{H}\left(f_{157}\right)\right)_{\infty} \subseteq T_{1}+T_{3}+\sum_{\alpha=5}^{8} T_{\alpha}$ and $T_{2}+T_{4} \subset\left(R_{H}\left(f_{258}\right)\right)_{\infty} \subseteq T_{2}+T_{4}+\sum_{\alpha=5}^{8} T_{\alpha}$ suffice for the linear independence of $1, R_{H}\left(f_{56}\right), R_{H}\left(f_{157}\right), R_{H}\left(f_{258}\right)$.

Further, observe that $H_{2}^{\theta}(n, 0)=\left\langle\tau_{33}^{n} \theta\right\rangle$ are subgroups of $H_{2 \times 2}^{\theta}(0)=\left\langle\tau_{33}, \theta\right\rangle$ with $\operatorname{rk} \Phi^{H_{2 \times 2}^{\theta}(0)}=2$. Therefore $\operatorname{rk} \Phi^{H_{2}^{\theta}(n, 0)}=2$ by Lemma $\left.11 \mathrm{iv}\right)$.

If $H=H_{2}^{\theta}(0,0)=\langle\theta\rangle$ then

$$
\begin{gathered}
R_{H}\left(f_{56}\right)=f_{56}+f_{78}, \quad R_{H}\left(f_{157}\right)=f_{157}-\mathrm{e}^{\frac{\pi}{2}} f_{357}, \quad R_{H}\left(f_{368}\right)=f_{368}-\mathrm{e}^{\frac{\pi}{2}} f_{168} \\
R_{H}\left(f_{258}\right)=f_{258}+f_{267}, \quad R_{H}\left(f_{467}\right)=f_{467}+f_{458} .
\end{gathered}
$$

The $\Gamma_{2}^{\theta}(0,0)$-cusps are $\bar{\kappa}_{1}=\bar{\kappa}_{3}, \bar{\kappa}_{2}, \bar{\kappa}_{4}, \bar{\kappa}_{5}=\bar{\kappa}_{7}$ and $\bar{\kappa}_{6}=\bar{\kappa}_{8}$. According to Lemma 11 ii), $T_{1} \subset\left(R_{H}\left(f_{157}\right)\right)_{\infty},\left(R_{H}\left(f_{168}\right)\right)_{\infty} \subseteq T_{1}+T_{3}+\sum_{\alpha=5}^{8} T_{\alpha}$ implies $R\left(f_{168}\right) \in \operatorname{Span}_{\mathbb{C}}\left(1, R_{H}\left(f_{56}\right), R\left(f_{157}\right)\right)$. Lemma 13 supplies $\left.\frac{f_{258}+f_{267}}{\Sigma_{2}}\right|_{T_{2}}=$ 
$2 \mathrm{e}^{-\pi} \neq 0$ and $\left.\frac{f_{467}+f_{458}}{\Sigma_{4}}\right|_{T_{4}}=0$. Therefore $\left.R_{H}\left(f_{258}\right)\right|_{T_{2}}=\infty$ and $R_{H}\left(f_{467}\right) \subset$ $\operatorname{Span}_{\mathbb{C}}\left(1, R_{H}\left(f_{56}\right)\right)$. Thus, $\mathcal{L}^{H}=\operatorname{Span}_{\mathbb{C}}\left(1, R_{H}\left(f_{56}\right), R_{H}\left(f_{157}\right), R_{H}\left(f_{258}\right)\right) \simeq$ $\mathbb{C}^{4}$. The entire $\left[\Gamma_{2}^{\theta}(0,0), 1\right]$ vanishes at $\bar{\kappa}_{4}$ and $\Phi^{H_{2}^{\theta}(0,0)}$ is not globally defined.

For $H=H_{2}^{\theta}(1,0)=\left\langle\tau_{33} \theta\right\rangle$ the space $\mathcal{L}^{H}$ is generated by

$$
\begin{array}{cl}
1 \in \mathbb{C}, & R_{H}\left(f_{56}\right)=f_{56}-f_{78} \\
R_{H}\left(f_{157}\right)=f_{157}+\mathrm{i} f_{368}, \quad R_{H}\left(f_{258}\right)=2 f_{258}, \quad R_{H}\left(f_{467}\right)=0 .
\end{array}
$$

The $\Gamma_{2}^{\theta}(1,0)$-cusps are $\bar{\kappa}_{1}=\bar{\kappa}_{3}, \bar{\kappa}_{2}, \bar{\kappa}_{4}, \bar{\kappa}_{5}=\bar{\kappa}_{8}$ and $\bar{\kappa}_{6}=\bar{\kappa}_{7}$. Making use of $T_{1} \subset\left(R_{H}\left(f_{157}\right)\right)_{\infty} \subseteq T_{1}+T_{3}+\sum_{\alpha=5}^{8} T_{\alpha}$ and $T_{2} \subset\left(R_{H}\left(f_{258}\right)\right)_{\infty} \subset T_{2}+\sum_{\alpha=5}^{8} T_{\alpha}$, one applies Lemma 11 iii), in order to conclude that

$$
\mathcal{L}^{H}=\operatorname{Span}_{\mathbb{C}}\left(1, R_{H}\left(f_{56}\right), R_{H}\left(f_{157}\right), R_{H}\left(f_{258}\right)\right) \simeq \mathbb{C}^{4}
$$

The abelian functions from $\mathcal{L}^{H}$ have no poles along $T_{4}$, so that $\Phi^{H_{2}^{\theta}(1,0)}$ is not defined at $\bar{\kappa}_{4}$.

Observe that $H_{2}^{\theta}(n, 1)=\left\langle\tau_{33}^{n} I J^{-1} \theta\right\rangle$ are subgroups of $H_{2 \times 2}^{\theta}(1)=\left\langle\tau_{33}, I J^{-1} \theta\right\rangle$ with $\operatorname{rk} \Phi^{H_{2 \times 2}^{\theta}(1)}=2$, so that $\operatorname{rk} \Phi^{H_{2}^{\theta}(n, 1)}=2$ as well.

More precisely, Reynolds operators for $H=H_{2}^{\theta}(0,1)=\left\langle I J^{-1} \theta\right\rangle$ are

$$
\begin{gathered}
R_{H}\left(f_{56}\right)=f_{56}+\mathrm{i} f_{78}, \quad R_{H}\left(f_{157}\right)=f_{157}-\mathrm{ie}^{\frac{\pi}{2}} f_{168}, \quad R_{H}\left(f_{368}\right)=f_{368}-\mathrm{ie}^{\frac{\pi}{2}} f_{357} \\
R_{H}\left(f_{258}\right)=f_{258}-\mathrm{e}^{-\frac{\pi}{2}} f_{458}, \quad R_{H}\left(f_{267}\right)=f_{267}+\mathrm{e}^{-\frac{\pi}{2}} f_{467} .
\end{gathered}
$$

The $\Gamma_{2}^{\theta}$-cusps are $\bar{\kappa}_{1}, \bar{\kappa}_{3}, \bar{\kappa}_{2}=\bar{\kappa}_{4}, \bar{\kappa}_{5}=\bar{\kappa}_{8}, \bar{\kappa}_{6}=\bar{\kappa}_{7}$. By Lemma 13 one has $\left.\frac{f_{157}-\mathrm{ie}^{\frac{\pi}{2}} f_{168}}{\Sigma_{1}}\right|_{T_{1}}=-2 \mathrm{ie}^{-\frac{\pi}{2}} \neq 0,\left.\frac{f_{368}-\mathrm{ie}^{\frac{\pi}{2}} f_{357}}{\Sigma_{3}}\right|_{T_{3}}=0$, whereas $\left.R_{H}\left(f_{157}\right)\right|_{T_{1}}=\infty$, $R_{H}\left(f_{368}\right) \in \operatorname{Span}_{\mathbb{C}}\left(1, R_{H}\left(f_{56}\right)\right)$. Applying Lemma 11 ii) to the inclusions $T_{2} \subset$ $\left(R_{H}\left(f_{258}\right)\right)_{\infty},\left(R_{H}\left(f_{267}\right)\right)_{\infty} \subseteq T_{2}+T_{4}+\sum_{\alpha=5}^{8} T_{\alpha}$, one concludes that $R_{H}\left(f_{267}\right) \in$ $\operatorname{Span}_{\mathbb{C}}\left(1, R_{H}\left(f_{56}\right), R_{H}\left(f_{258}\right)\right)$. Altogether

$$
\mathcal{L}^{H}=\operatorname{Span}_{\mathbb{C}}\left(1, R_{H}\left(f_{56}\right), R_{H}\left(f_{157}\right), R_{H}\left(f_{258}\right)\right) \simeq \mathbb{C}^{4} .
$$

Since $\mathcal{L}^{H}$ has no pole over $T_{3}$, the rational map $\Phi^{H_{2}^{\theta}(0,1)}$ is not defined at $\bar{\kappa}_{3}$. If $H=H_{2}^{\theta}(1,1)=\left\langle\tau_{33} I J^{-1} \theta\right\rangle$ then

$$
\begin{gathered}
R_{H}\left(f_{56}\right)=f_{56}-\mathrm{i} f_{78}, \quad R_{H}\left(f_{157}\right)=2 f_{157} \\
R_{H}\left(f_{368}\right)=0, \quad R_{H}\left(f_{258}\right)=f_{258}+\mathrm{e}^{-\frac{\pi}{2}} f_{467} .
\end{gathered}
$$


The $\Gamma_{2}^{\theta}(1,1)$-cusps are $\bar{\kappa}_{1}, \bar{\kappa}_{3}, \bar{\kappa}_{2}=\bar{\kappa}_{4}, \bar{\kappa}_{5}=\bar{\kappa}_{7}$ and $\bar{\kappa}_{6}=\bar{\kappa}_{8}$. Making use of $\left.R_{H}\left(f_{157}\right)\right|_{T_{1}}=\infty,\left.T_{H}\left(f_{258}\right)\right|_{T_{2}}=\infty$, one applies Lemma 11 iii), in order to conclude that $\mathcal{L}^{H}=\operatorname{Span}_{\mathbb{C}}\left(1, R_{H}\left(f_{56}\right), R_{H}\left(f_{157}\right), R_{H}\left(f_{258}\right)\right) \simeq \mathbb{C}^{4}$. Since $\mathcal{L}^{H}$ has no pole over $T_{3}$, the rational map $\Phi^{H_{2}^{\theta}(1,1)}$ is not defined at $\bar{\kappa}_{3}$.

Reynolds operators for $H=H_{2}^{\theta}(0,2)=\left\langle I^{2} J^{2} \theta\right\rangle$ are

$$
\begin{gathered}
R_{H}\left(f_{56}\right)=f_{56}-f_{78}, \quad R_{H}\left(f_{157}\right)=f_{157}+\mathrm{e}^{\frac{\pi}{2}} f_{357}, \quad R_{H}\left(f_{168}\right)=f_{168}+\mathrm{e}^{-\frac{\pi}{2}} f_{368} \\
R_{H}\left(f_{258}\right)=f_{258}-f_{267}, \quad R_{H}\left(f_{467}\right)=f_{467}-f_{458} .
\end{gathered}
$$

The $\Gamma_{2}^{\theta}(0,2)$-cusps are $\bar{\kappa}_{1}=\bar{\kappa}_{3}, \bar{\kappa}_{2}, \bar{\kappa}_{4}, \bar{\kappa}_{5}=\bar{\kappa}_{7}, \bar{\kappa}_{6}=\bar{\kappa}_{8}$. Lemma 11 ii) applies to $T_{1} \subset\left(R_{H}\left(f_{157}\right)\right)_{\infty},\left(R_{H}\left(f_{168}\right)\right)_{\infty} \subseteq T_{1}+T_{3}+\sum_{\alpha=5}^{8} T_{\alpha}$ to provide $R_{H}\left(f_{168}\right) \in$ $\operatorname{Span}_{\mathbb{C}}\left(1, R_{H}\left(f_{56}\right), R_{H}\left(f_{157}\right)\right)$. By Lemma 13 one has $\left.\frac{f_{258}-f_{267}}{\Sigma_{2}}\right|_{T_{2}}=0$ and $\left.\frac{f_{467}-f_{458}}{\Sigma_{4}}\right|_{T_{4}}=2 \mathrm{ie}^{-\frac{\pi}{2}} \neq 0$. As a result, $R_{H}\left(f_{258}\right) \in \operatorname{Span}_{\mathbb{C}}\left(1, R_{H}\left(f_{56}\right)\right)$ and $\left.R_{H}\left(f_{467}\right)\right|_{T_{4}}=\infty$. Lemma 11 iii) reveals that $1 \in \mathbb{C}, R_{H}\left(f_{56}\right), R_{H}\left(f_{157}\right)$, $R_{H}\left(f_{467}\right)$ form a $\mathbb{C}$-basis of $\mathcal{L}^{H}$. Since $\mathcal{L}^{H}$ has no pole over $T_{2}$, the rational map $\Phi^{H_{2}^{\theta}(0,2)}$ is not defined over $\bar{\kappa}_{2}$.

In the case of $H=H_{2}^{\theta}(1,2)=\left\langle\tau_{33} I^{2} J^{2} \theta\right\rangle$ one has

$$
\begin{array}{cc}
R_{H}\left(f_{56}\right)=f_{56}+f_{78}, & R_{H}\left(f_{157}\right)=f_{157}-\mathrm{i} f_{368} \\
R_{H}\left(f_{258}\right)=0, & R_{H}\left(f_{467}\right)=2 f_{467} .
\end{array}
$$

The $\Gamma_{2}^{\theta}(1,2)$-cusps are $\bar{\kappa}_{1}=\bar{\kappa}_{3}, \bar{\kappa}_{2}, \bar{\kappa}_{4}, \bar{\kappa}_{5}=\bar{\kappa}_{8}$ and $\bar{\kappa}_{6}=\bar{\kappa}_{7}$. Lemma 11 iii) applies to $T_{1} \subset\left(R_{H}\left(f_{157}\right)\right)_{\infty} \subseteq T_{1}+T_{3}+\sum_{\alpha=5}^{8} T_{\alpha}, T_{4} \subset\left(R_{H}\left(f_{467}\right)\right)_{\infty} \subseteq$ $T_{4}+T_{6}+T_{7}$, in order to justify the linear independence of $1, R_{H}\left(f_{56}\right), R_{H}\left(f_{157}\right)$, $R_{H}\left(f_{467}\right)$. Since $\mathcal{L}^{H} \simeq \mathbb{C}^{4}$ has no pole over $T_{2}$, the rational map $\Phi^{H_{2}^{\theta}(1,2)}$ is not defined at $\bar{\kappa}_{2}$.

ii) For $H=H_{2}(0,0)=\left\langle\tau_{33}\right\rangle$ one has the following Reynolds operators

$$
R_{H}\left(f_{56}\right)=0, \quad R_{H}\left(f_{78}\right)=0, \quad R_{H}\left(f_{157}\right)=f_{157}-\mathrm{ie}^{\frac{\pi}{2}} f_{168}
$$

$R_{H}\left(f_{258}\right)=f_{258}+f_{267}, \quad R_{H}\left(f_{368}\right)=f_{368}+\mathrm{ie}^{\frac{\pi}{2}} f_{357}, \quad R_{H}\left(f_{467}\right)=f_{467}-f_{458}$.

There are six $\Gamma_{\left\langle\tau_{33}\right\rangle}$-cusps: $\bar{\kappa}_{1}, \bar{\kappa}_{2}, \bar{\kappa}_{3}, \bar{\kappa}_{4}, \bar{\kappa}_{5}=\bar{\kappa}_{6}$ and $\bar{\kappa}_{7}=\bar{\kappa}_{8}$. By the means of Lemma 13 one observes that $\left.\frac{f_{157}-\mathrm{ie}^{\frac{\pi}{2}} f_{168}}{\Sigma_{1}}\right|_{T_{1}}=-2 \mathrm{ie}^{-\frac{\pi}{2}} \neq 0,\left.\frac{f_{258}+f_{267}}{\Sigma_{2}}\right|_{T_{2}}=$ $2 \mathrm{e}^{-\pi} \neq 0,\left.\frac{f_{368}+\mathrm{ie}^{\frac{\pi}{2}} f_{357}}{\Sigma_{3}}\right|_{T_{3}}=2 \mathrm{ie}^{-\frac{\pi}{2}} \neq 0,\left.\frac{f_{467}-f_{458}}{\Sigma_{4}}\right|_{T_{4}}=2 \mathrm{ie}^{-\frac{\pi}{2}} \neq 0$. Therefore 
$T_{i} \subset\left(R_{H}\left(f_{i, \alpha_{i}, \beta_{i}}\right)\right)_{\infty} \subseteq T_{i}+\sum_{\delta=5}^{8} T_{\delta}$ for $1 \leq i \leq 4,\left(\alpha_{1}, \beta_{1}\right)=(5,7),\left(\alpha_{2}, \beta_{2}\right)=$ $(5,8),\left(\alpha_{3}, \beta_{3}\right)=(6,8),\left(\alpha_{4}, \beta_{4}\right)=(6,7)$. According to Lemma 11 iii), that suffices for $1, R_{H}\left(f_{157}\right), R_{H}\left(f_{258}\right), R_{H}\left(f_{368}\right), R_{H}\left(f_{467}\right)$ to be a $\mathbb{C}$-basis of $\mathcal{L}^{H}$. Bearing in mind that $H_{2}(0,0)=\left\langle\tau_{33}\right\rangle$ is a subgroup of $H_{2 \times 2}^{\prime}(0)=\left\langle\tau_{33}, I^{2}\right\rangle$ with $\operatorname{rk} \Phi^{H_{2 \times 2}^{\prime}(0)}=2$, one concludes that $\operatorname{rk} \Phi^{\left\langle\tau_{33}\right\rangle}=2$.

\section{References}

[1] Hacon Ch. and Pardini R., Surfaces with $p_{g}=q=3$, Trans. Amer. Math. Soc. 354 (2002) 2631-1638.

[2] Hemperly J., The Parabolic Contribution to the Number of Independent Automorphic Forms on a Certain Bounded Domain, Amer. J. Math. 94 (1972) 1078-1100.

[3] Holzapfel R.-P., Jacobi Theta Embedding of a Hyperbolic 4-space with Cusps, In: Geometry, Integrability and Quantization IV, I. Mladenov and G. Naber (Eds), Coral Press, Sofia 2002, pp 11-63.

[4] Holzapfel R.-P., Complex Hyperbolic Surfaces of Abelian Type, Serdica Math. J. 30 (2004) 207-238.

[5] Kasparian A. and Kotzev B., Normally Generated Subspaces of Logarithmic Canonical Sections, to appear in Ann. Univ. Sofia.

[6] Kasparian A. and Kotzev B., Weak Form of Holzapfel's Conjecture, J. Geom. Symm. Phys. 19 (2010) 29-42.

[7] Kasparian A. and Nikolova L., Ball Quotients of Non-Positive Kodaira Dimension, submitted to CRAS (Sofia).

[8] Lang S., Elliptic Functions, Addison-Wesley, London 1973, pp 233-237.

[9] Momot A., Irregular Ball-Quotient Surfaces with Non-Positive Kodaira Dimension, Math. Res. Lett. 15 (2008) 1187-1195.

Azniv Kasparian

Department of Mathematics

and Informatics

Sofia University "Kliment Ohridski"

Sofia 1164, BULGARIA

E-mail address: kasparia@fmi.uni-sofia.bg 